\title{
A Structure-Property Study Toward $\pi$-Extended Phosphole Chromophores with Ambipolar Redox Properties
}

\author{
Supporting Information
}

\author{
Yi Ren ${ }^{\dagger}$ and Thomas Baumgartner* \\ Department of Chemistry and Centre for Advanced Solar Materials, University of \\ Calgary, 2500 University Drive NW, Calgary, AB, T2N 1N4, Canada \\ ${ }^{\dagger}$ Current address: Department of Chemical \& Biological Engineering, Princeton \\ University, Princeton, NJ 08544, USA \\ E-mail: thomas.baumgartner@ucalgary.ca
}


Figure S1. Frontier Orbitals for 3 (B3LYP/6-31+G(d) level of theory).

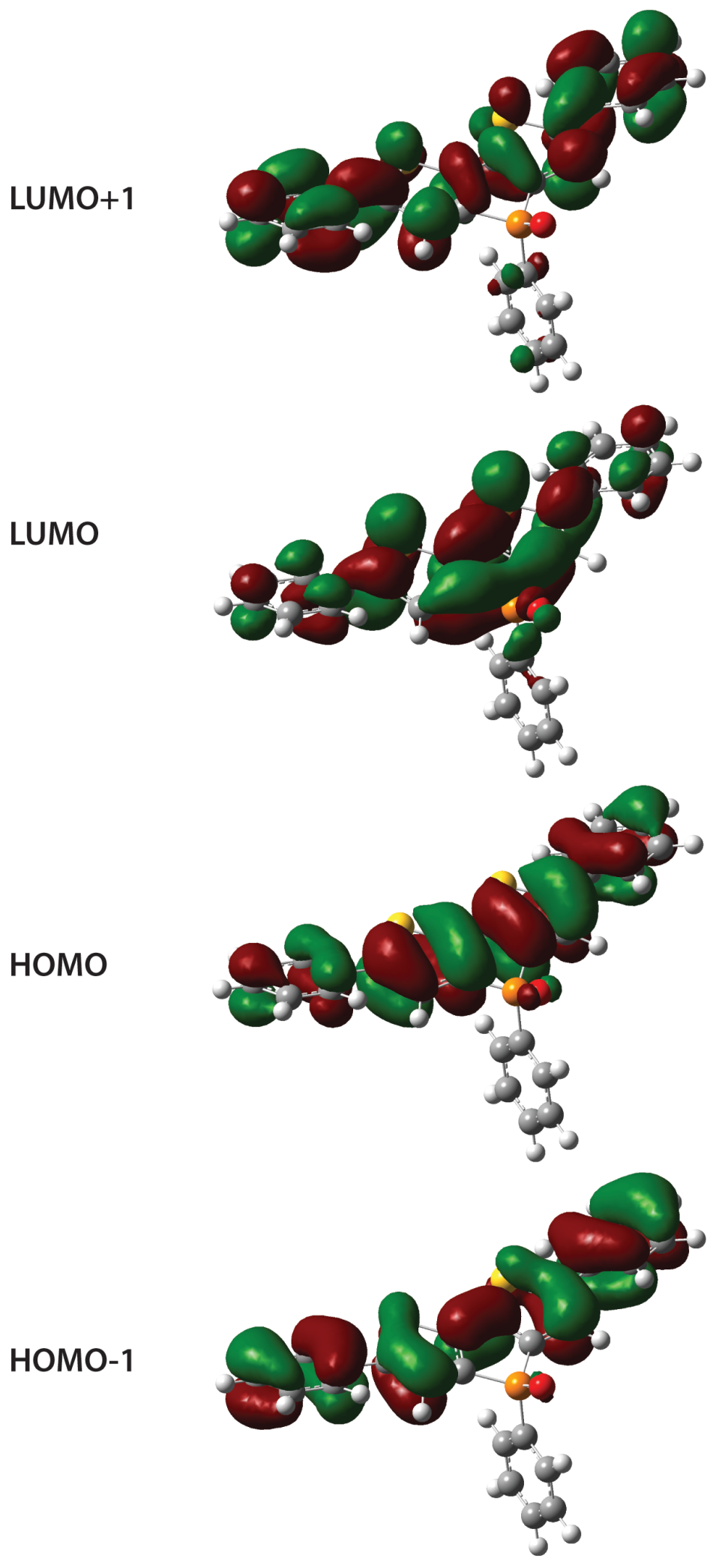


Figure S2. Frontier Orbitals for 4 (B3LYP/6-31+G(d) level of theory).

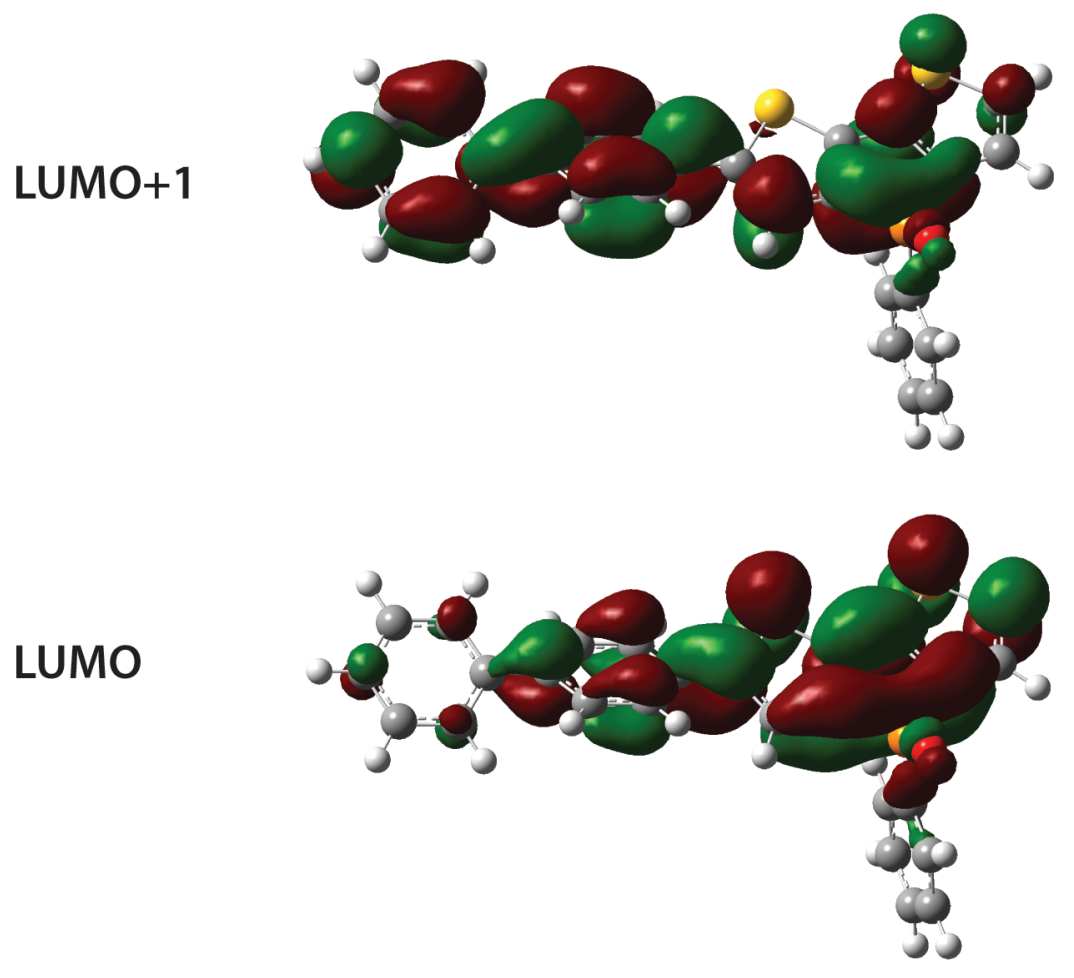

HOMO

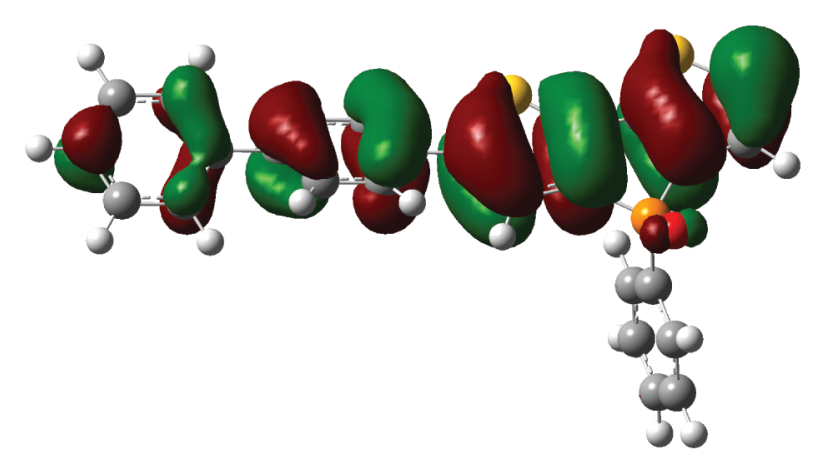

HOMO-1

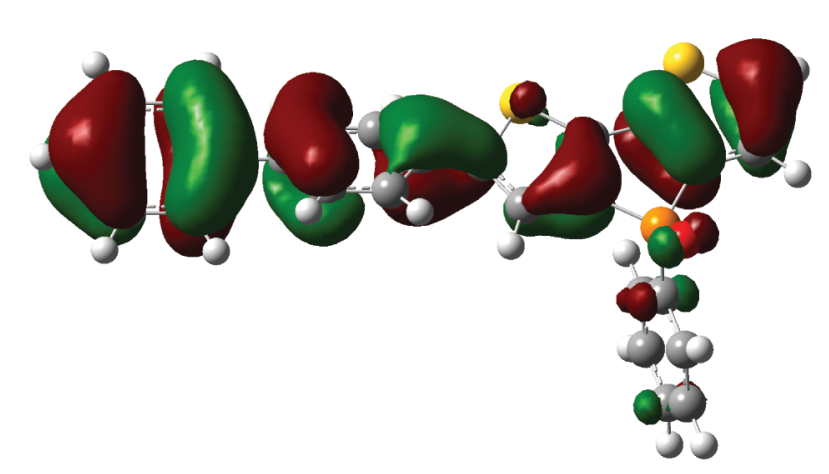


Figure S3. Frontier Orbitals for 5 (B3LYP/6-31+G(d) level of theory).

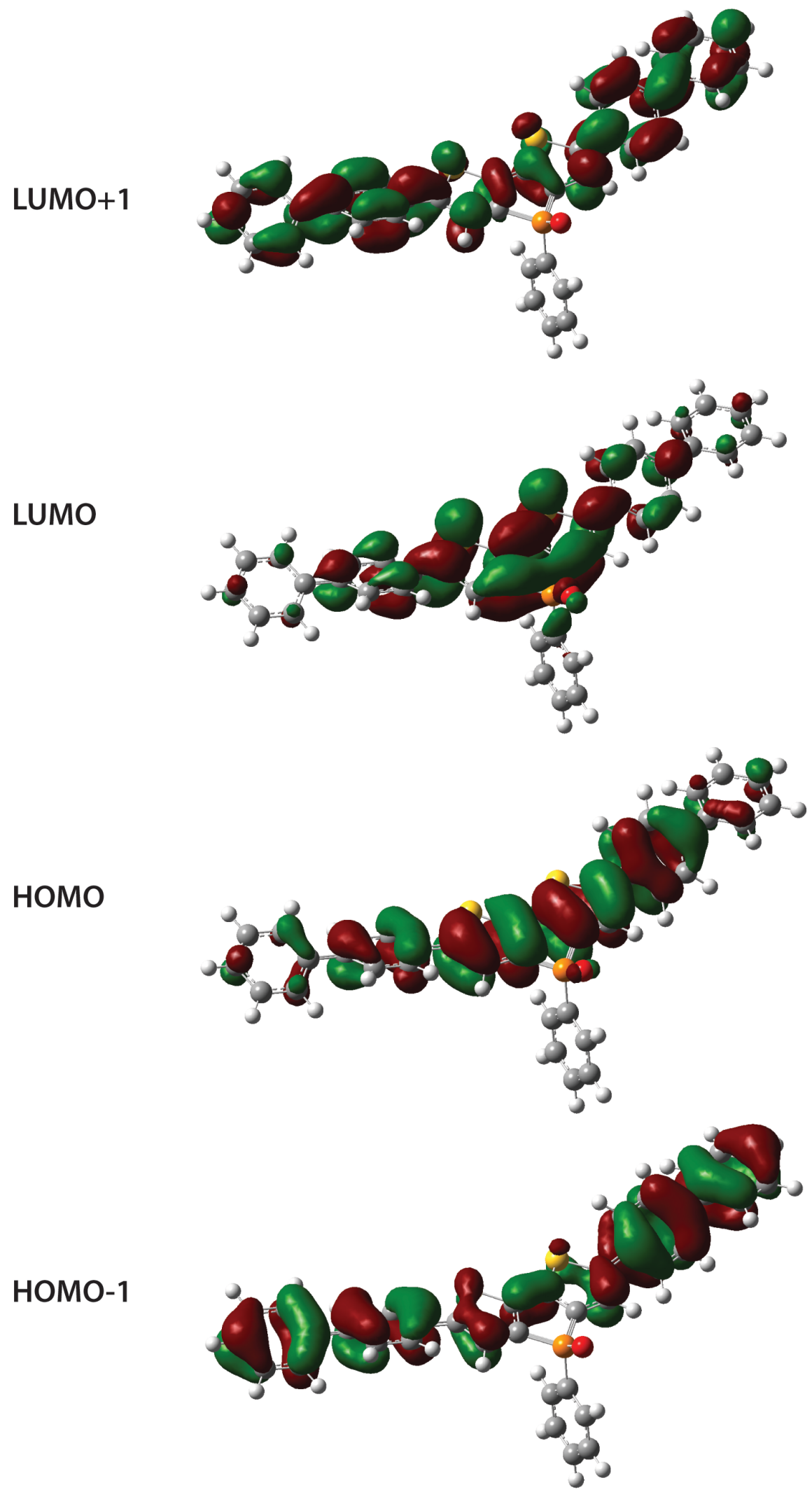


Figure S4. Frontier Orbitals for 6 (B3LYP/6-31+G(d) level of theory; $\mathrm{PCM}, \mathrm{CH}_{2} \mathrm{Cl}_{2}$ ).

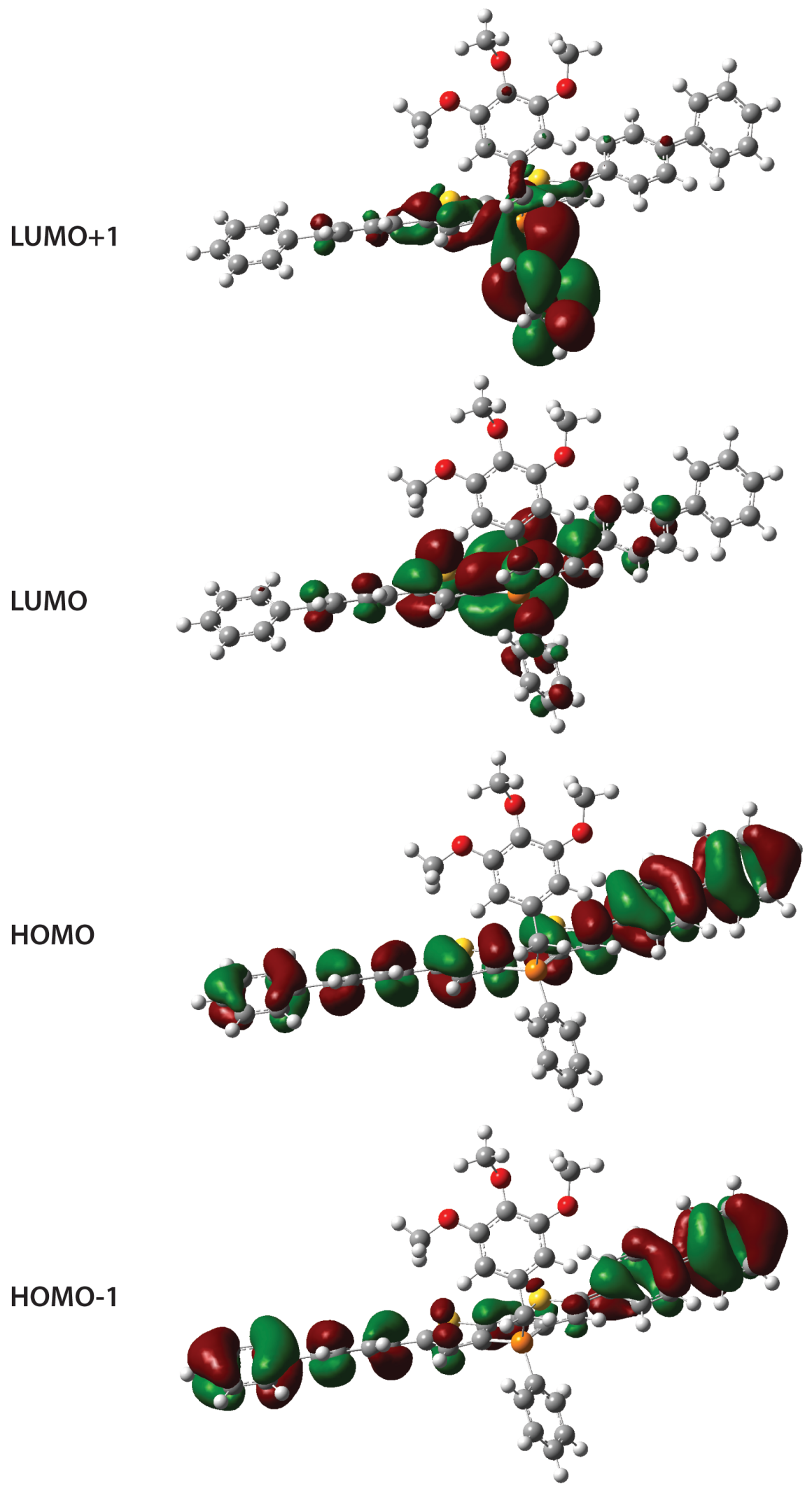


Figure S5. Frontier Orbitals for 3-ox (B3LYP/6-31+G(d) level of theory; PCM, $\mathrm{CH}_{2} \mathrm{Cl}_{2}$ ).
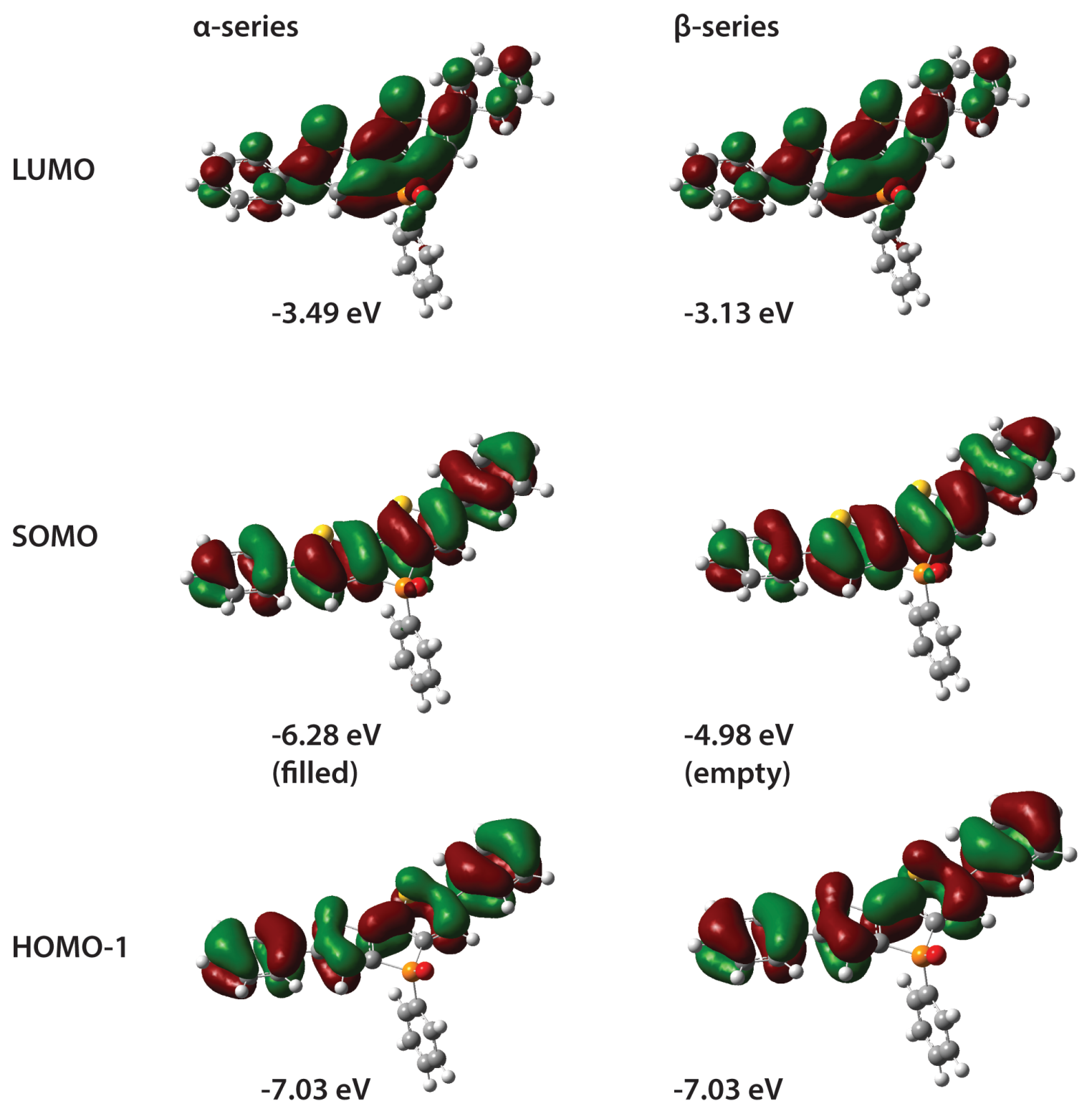
Figure S6. Frontier Orbitals for 4-ox (B3LYP/6-31+G(d) level of theory; PCM, $\mathrm{CH}_{2} \mathrm{Cl}_{2}$ ).

a-series

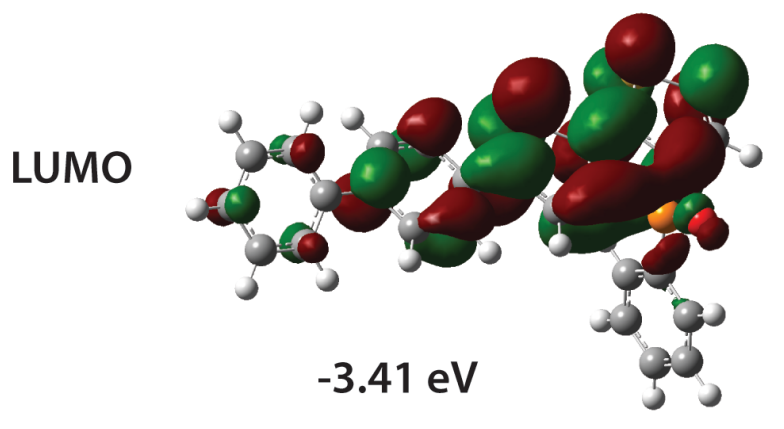

SOMO

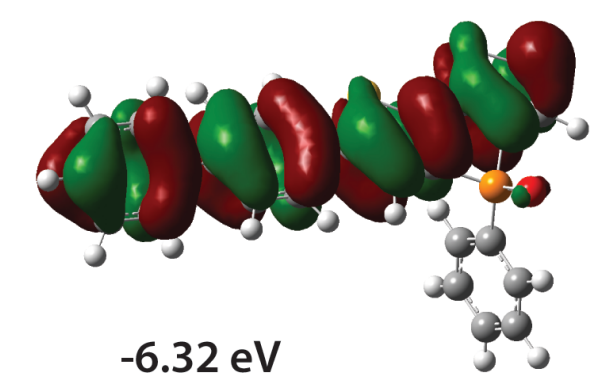

(filled)

HOMO-1

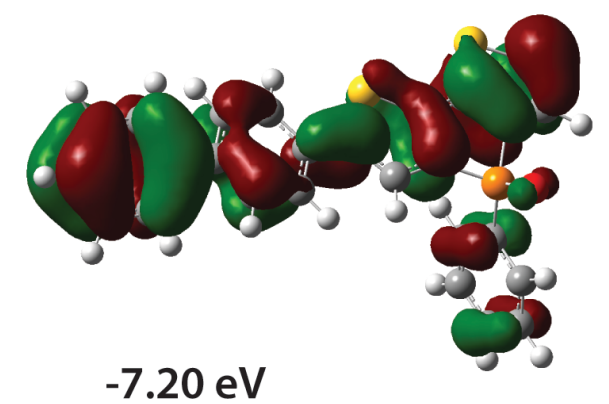

$\beta$-series
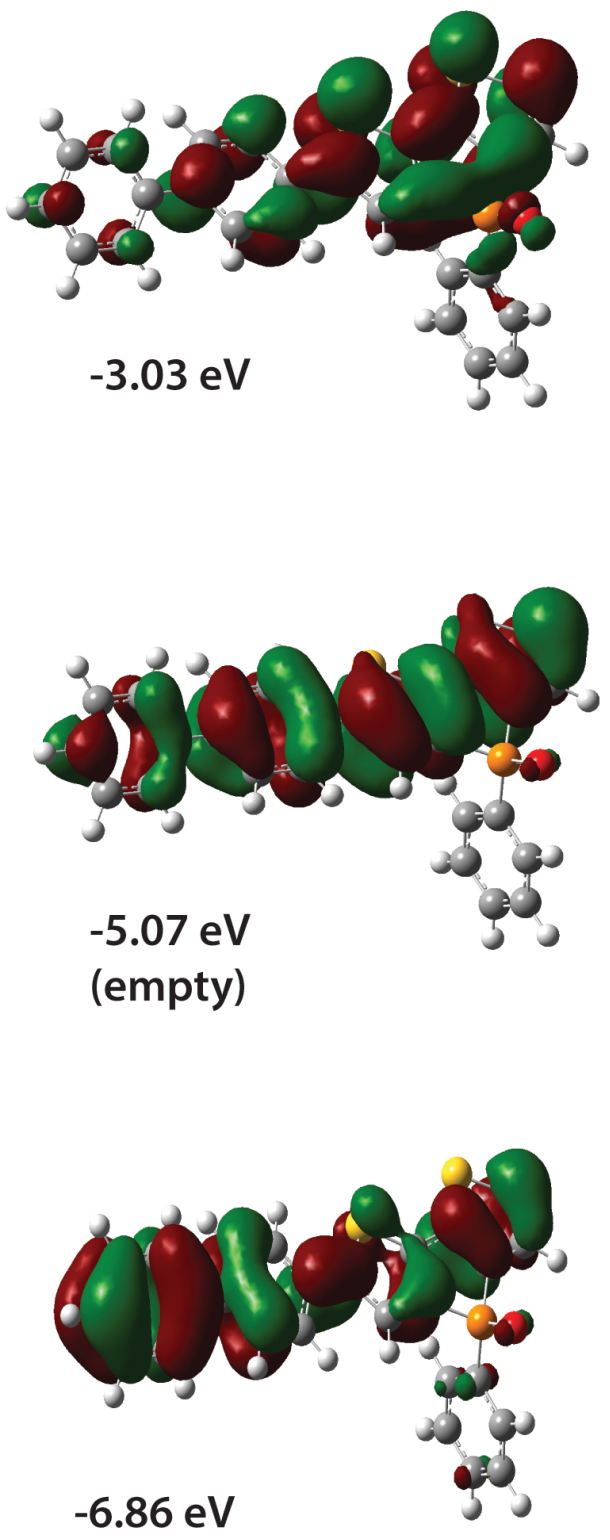
Figure S7. Frontier Orbitals for 5-ox (B3LYP/6-31+G(d) level of theory; PCM, $\mathrm{CH}_{2} \mathrm{Cl}_{2}$ ).
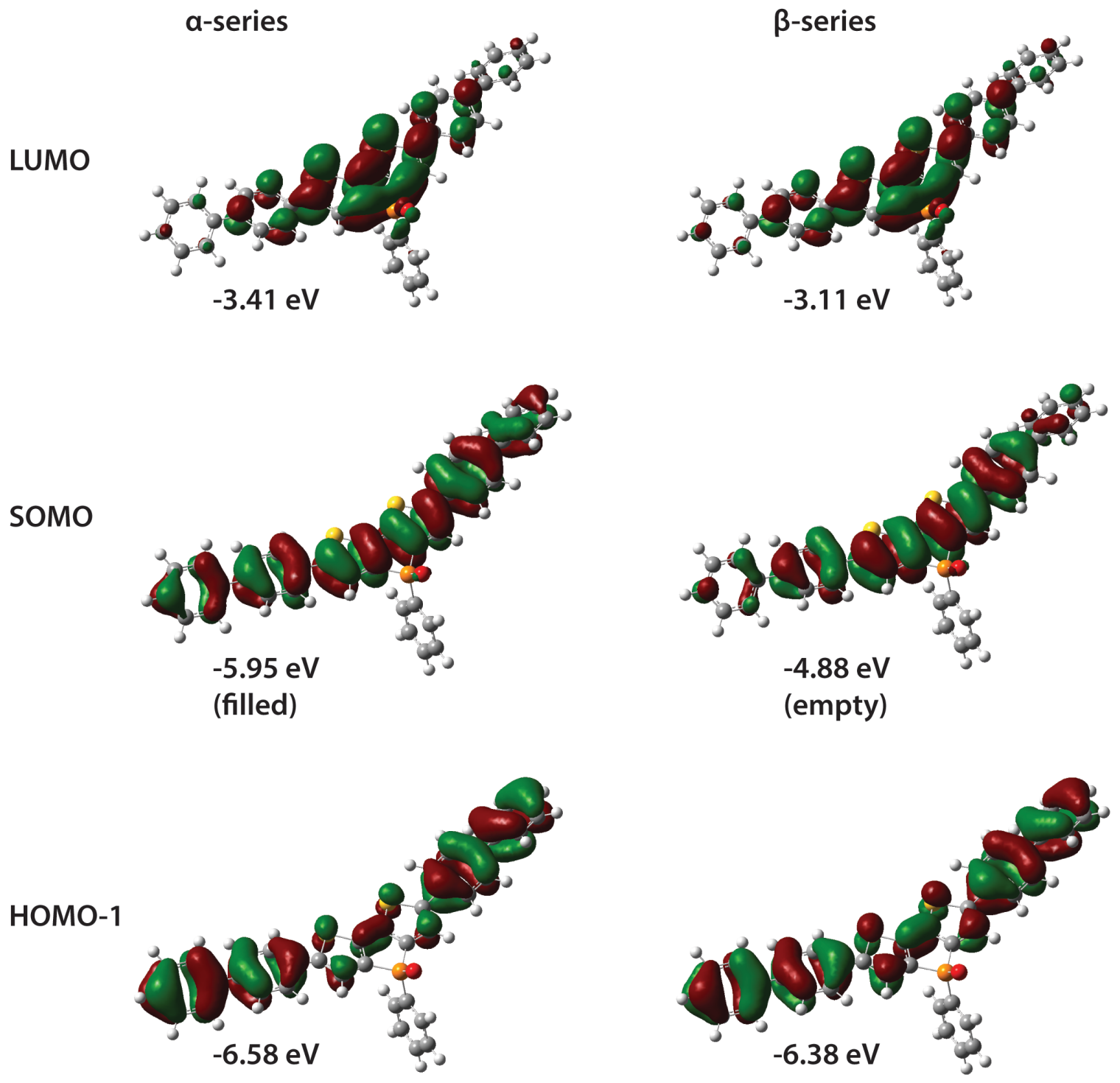
Figure S8. Frontier Orbitals for 5-ox2 (B3LYP/6-31+G(d) level of theory; PCM, $\mathrm{CH}_{2} \mathrm{Cl}_{2}$ ).
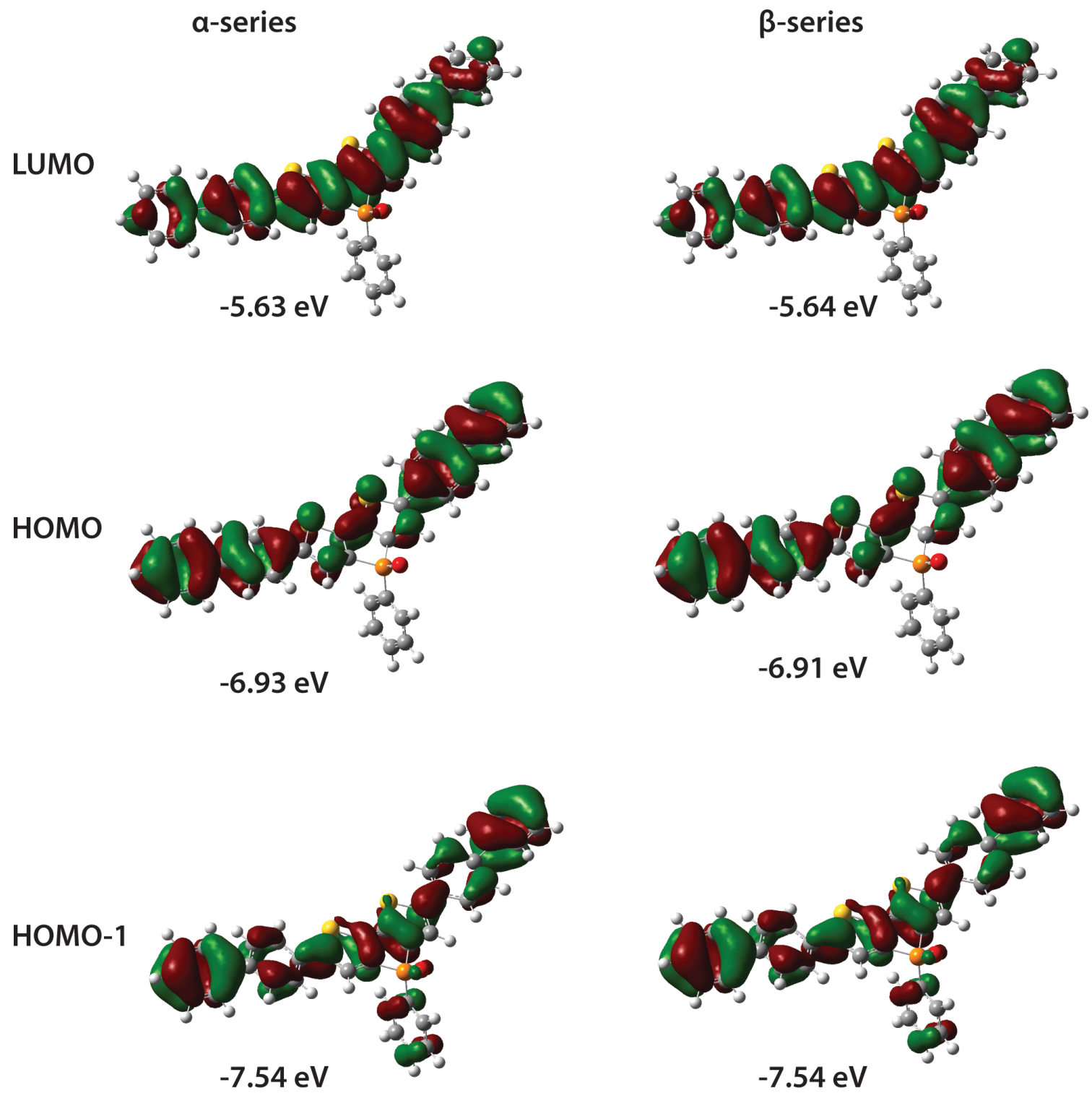
Figure S9. Frontier Orbitals for 3-red (B3LYP/6-31+G(d) level of theory; PCM, $\mathrm{CH}_{2} \mathrm{Cl}_{2}$ ).

$$
\text { a-series }
$$

LUMO

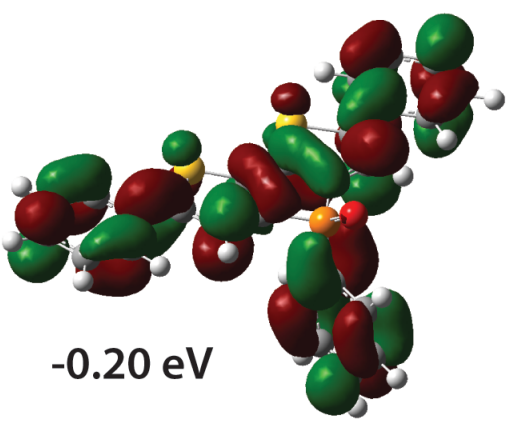

SOMO

HOMO-1

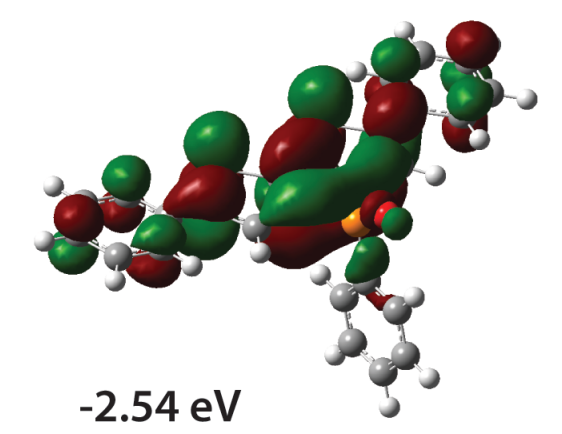

(filled)

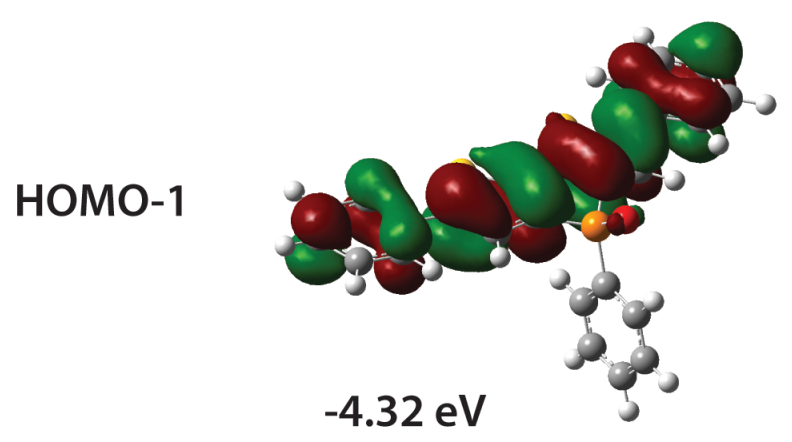

$\beta$-series
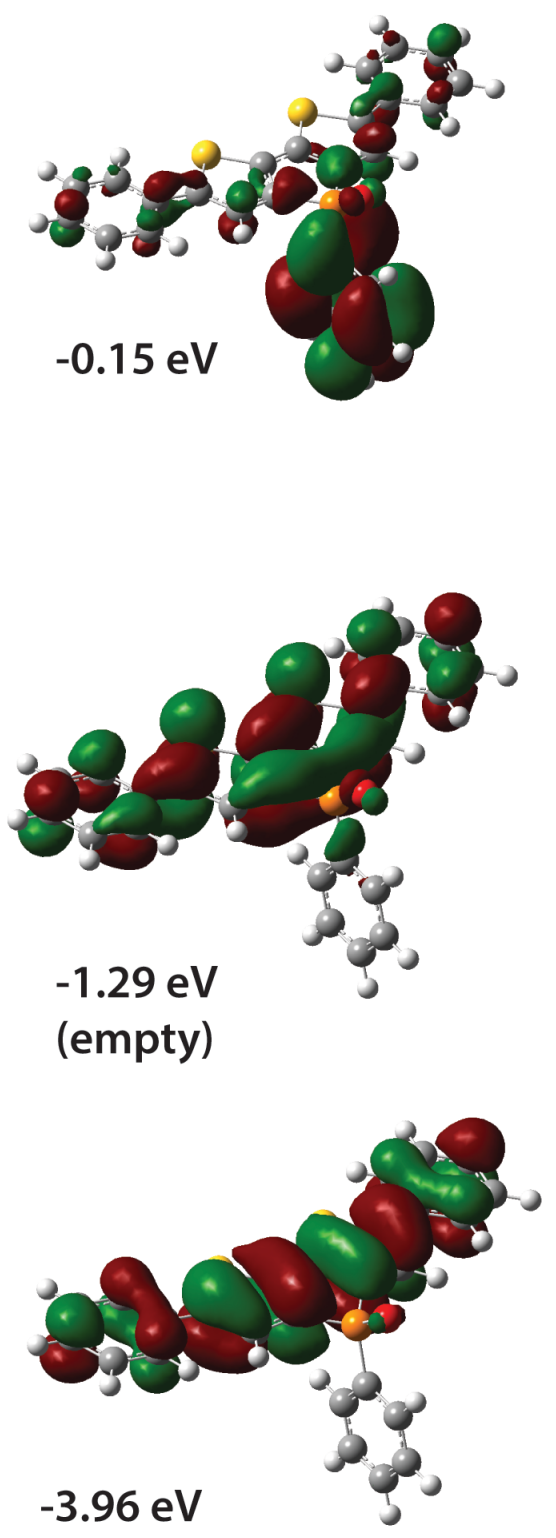
Figure S10. Frontier Orbitals for 4-red (B3LYP/6-31+G(d) level of theory; PCM, $\mathrm{CH}_{2} \mathrm{Cl}_{2}$ ).

$$
\text { a-series }
$$
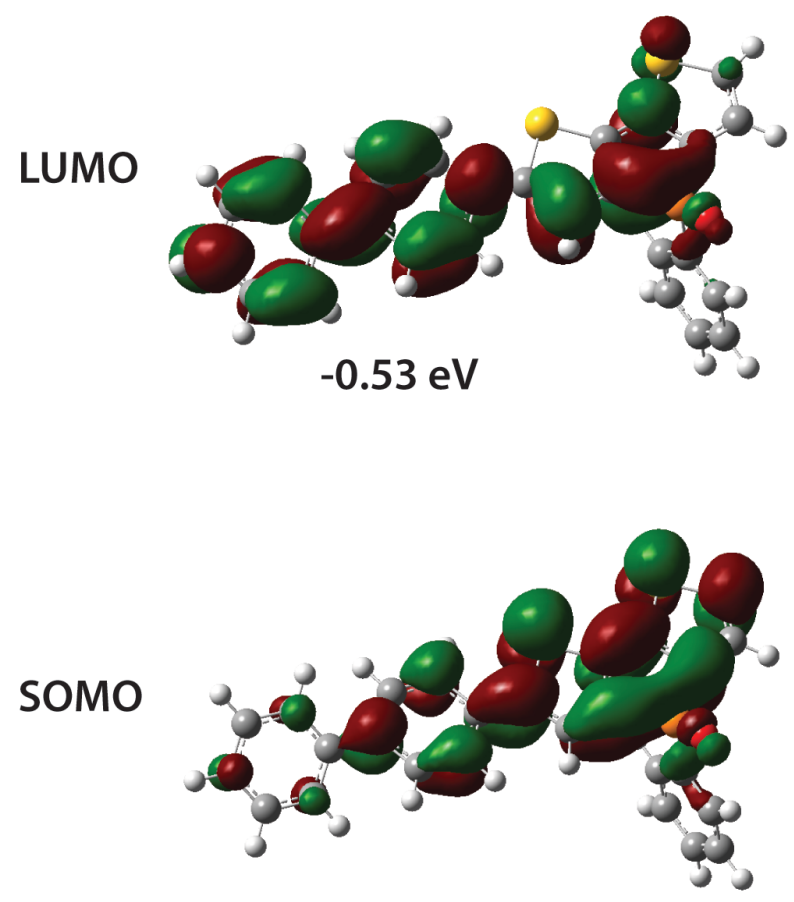

$-2.42 \mathrm{eV}$

(filled)

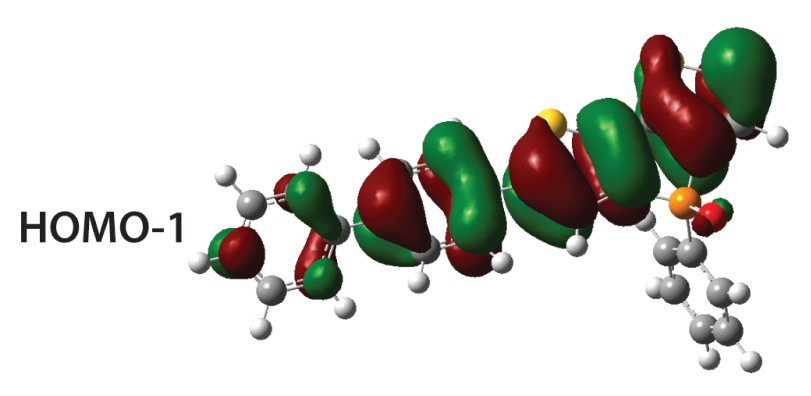

$-4.38 \mathrm{eV}$ $\beta$-series
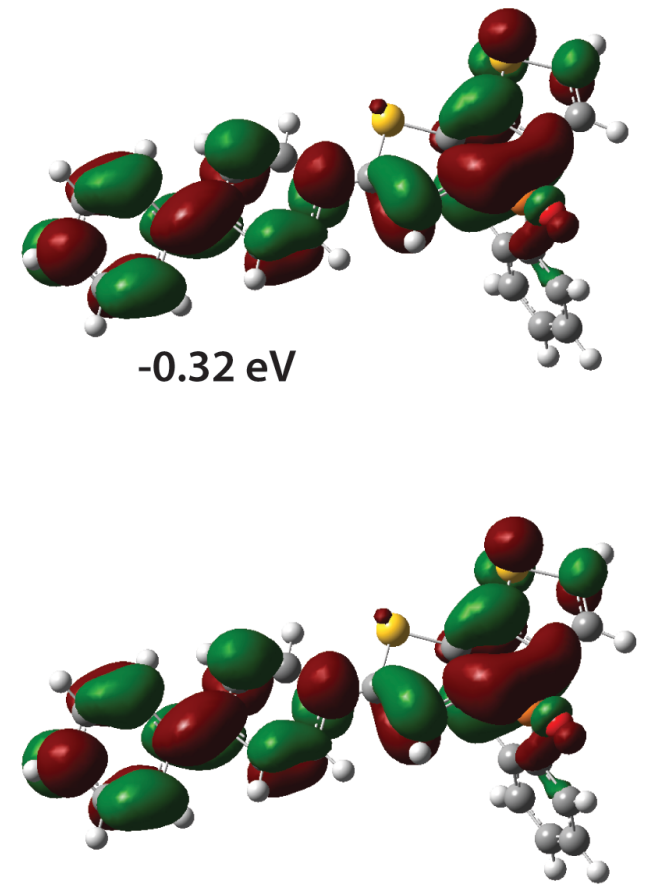

$-1.14 \mathrm{eV}$ (empty)

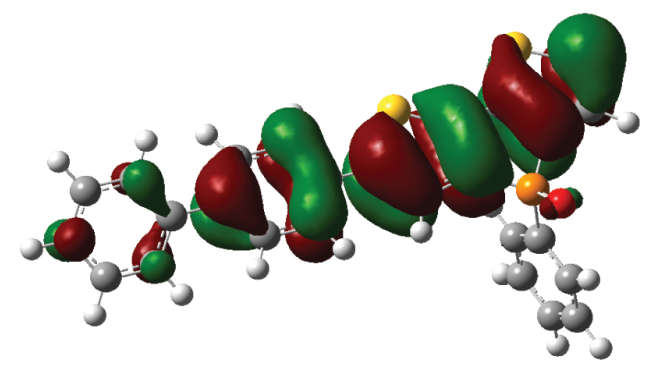

$-4.00 \mathrm{eV}$ 
Figure S11. Frontier Orbitals for 5-red (B3LYP/6-31+G(d) level of theory; PCM, $\mathrm{CH}_{2} \mathrm{Cl}_{2}$ ).
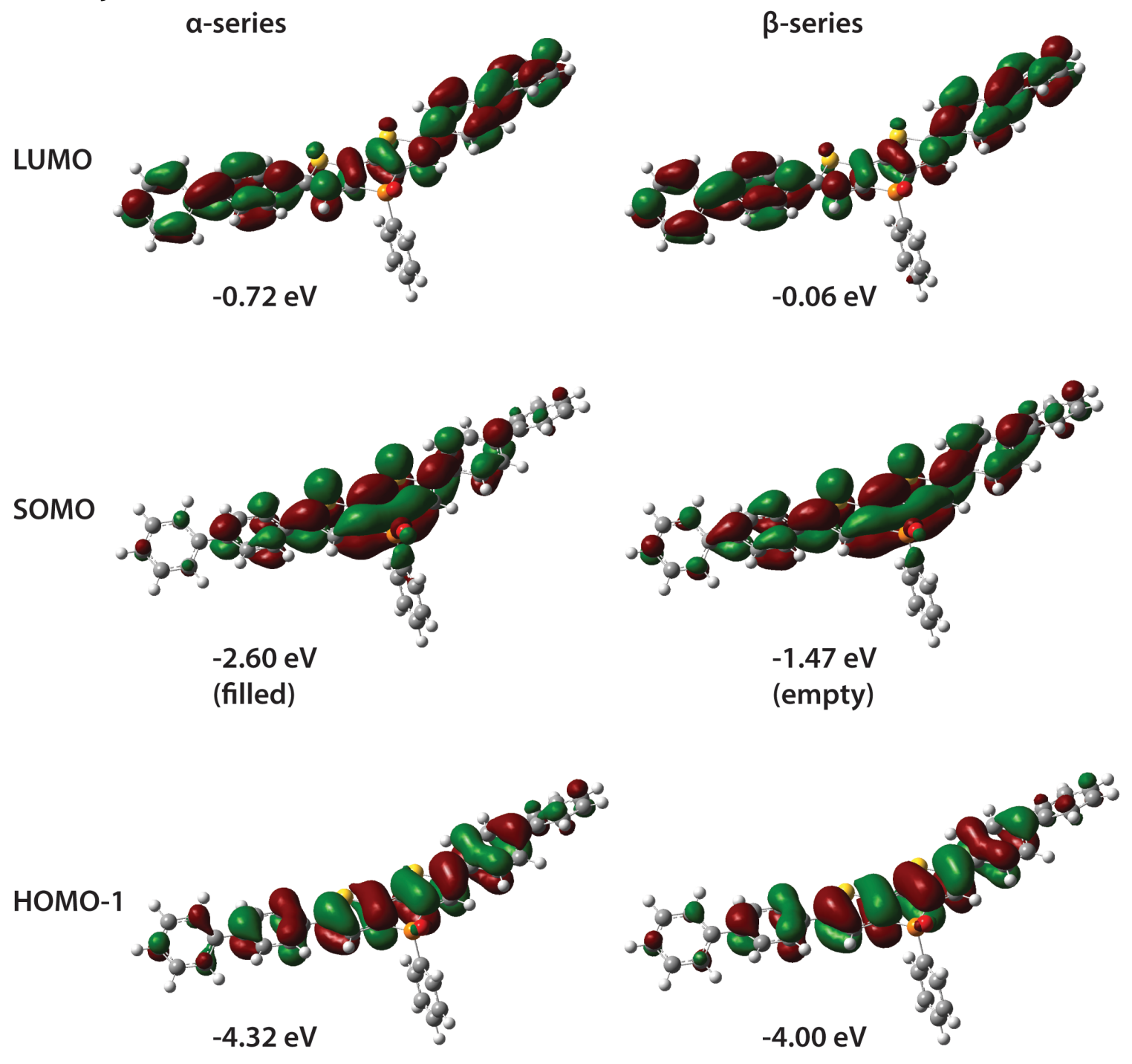
Table S1. Cartesian coordinates for minimized structure of compound 3.

\begin{tabular}{|c|c|c|c|c|c|}
\hline \multirow{2}{*}{$\begin{array}{l}\text { Center } \\
\text { Number }\end{array}$} & \multirow{2}{*}{\multicolumn{2}{|c|}{$\begin{array}{l}\text { Atomic } \\
\text { Number }\end{array}$}} & Atomic & \multicolumn{2}{|c|}{ Coordinates (Angstrom } \\
\hline & & & Type & $X$ & Z \\
\hline 1 & 15 & 0 & 0.000077 & 1.560695 & -1.173516 \\
\hline 2 & 6 & 0 & -1.304254 & 0.371059 & -0.677799 \\
\hline 3 & 6 & 0 & -0.724506 & -0.789900 & -0.192950 \\
\hline 4 & 6 & 0 & 0.724919 & -0.789691 & -0.192751 \\
\hline 5 & 6 & 0 & 1.304565 & 0.371475 & -0.677335 \\
\hline 6 & 6 & 0 & -2.718373 & 0.331508 & -0.664119 \\
\hline 7 & 6 & 0 & -3.226514 & -0.855434 & -0.172053 \\
\hline 8 & 16 & 0 & -1.915280 & -1.952840 & 0.2 \\
\hline 9 & 16 & 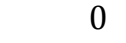 & $1.91 !$ & -1.952611 & 0.2 \\
\hline 10 & 6 & 0 & 3.226985 & -0.854937 & -0.171864 \\
\hline 11 & 6 & 0 & 2.71 & 0.332015 & -0.6 \\
\hline 12 & 6 & 0 & -4.629307 & -1.252198 & -0.016089 \\
\hline 13 & 6 & 0 & 4.629869 & -1.251488 & -0.015903 \\
\hline 14 & 6 & 0 & -5.616053 & -0.698624 & -0.854278 \\
\hline 15 & 6 & 0 & -6.955377 & -1.048591 & -0.705368 \\
\hline 16 & 6 & 0 & -7.340415 & -1.970152 & 0.271198 \\
\hline 17 & 6 & 0 & -6.370894 & -2.535562 & 1.101507 \\
\hline 18 & 6 & 0 & -5.031589 & -2.178104 & 0.964006 \\
\hline 19 & 6 & 0 & 5.032189 & -2.178011 & 0.963563 \\
\hline 20 & 6 & 0 & 6.371564 & -2.535318 & 1.101064 \\
\hline 21 & 6 & 0 & 7.341101 & -1.969033 & 0.2 \\
\hline 22 & 6 & 0 & & 891 & -0 \\
\hline 23 & 6 & 0 & 5.616736 & $-0.6^{\mathrm{C}}$ & \\
\hline 24 & 8 & 0 & 0.0004 & 2.075585 & -2.5 \\
\hline 25 & 6 & 0 & -0.000648 & 339 & 0.0 \\
\hline 26 & 6 & 0 & -0.001076 & 2.668 & 63 \\
\hline 27 & 6 & 0 & -0.001578 & 3.739754 & 2.335392 \\
\hline 28 & 6 & 0 & -0.001781 & 5.052071 & 1.852892 \\
\hline 29 & 6 & 0 & -0.001373 & 5.291979 & 0.478374 \\
\hline 30 & 6 & 0 & -0.000830 & 4.221640 & -0.418581 \\
\hline 31 & 1 & 0 & -3.352259 & 1.151846 & -0.981060 \\
\hline 32 & 1 & 0 & 3.352343 & 1.152607 & -0.980573 \\
\hline 33 & 1 & 0 & -5.324091 & -0.008408 & $3-1.640007$ \\
\hline 34 & 1 & 0 & -7.699469 & -0.609917 & -1.364616 \\
\hline 35 & 1 & 0 & -8 & -2.2 & 0 \\
\hline 36 & 1 & 0 & 3215 & -3.25 & 1.867198 \\
\hline 37 & 1 & 0 & -4.292709 & -2.6 & 1.637457 \\
\hline 38 & 1 & 0 & 4.293252 & -2.6 & 1.636 \\
\hline 39 & 1 & 0 & 6.658836 & -3.2 & 1.86 \\
\hline 40 & 1 & 0 & 8.385801 & -2.245784 & 0.381731 \\
\hline 41 & 1 & 0 & 7.700292 & -0.607476 & -1.363233 \\
\hline 42 & 1 & 0 & 5.324896 & -0.006416 & -1.638847 \\
\hline 43 & 1 & 0 & -0.000953 & 1.650367 & 1.823666 \\
\hline 44 & 1 & 0 & -0.001891 & 3.552779 & 3.405773 \\
\hline 45 & 1 & 0 & -0.002232 & 5.885634 & 2.550301 \\
\hline 46 & 1 & 0 & -0.001545 & 6.311553 & 0.102382 \\
\hline 47 & 1 & 0 & -0.000496 & 4.390803 & -1.491309 \\
\hline
\end{tabular}


Table S2. Cartesian coordinates for minimized structure of compound 4.

\begin{tabular}{|c|c|c|c|c|c|}
\hline \multirow{2}{*}{$\begin{array}{l}\text { Center } \\
\text { Number }\end{array}$} & \multicolumn{2}{|c|}{ Ator } & Atomic & \multicolumn{2}{|c|}{ Coordinates (Angstrom } \\
\hline & & Number & Type & $\mathrm{X}$ & $\mathrm{Z}$ \\
\hline 1 & 15 & 0 & -3.373315 & 0.473506 & 1.13397 \\
\hline 2 & ( & 0 & -1.698185 & -0.109764 & 0.668030 \\
\hline 3 & 6 & 0 & -1.780827 & -1.361435 & 0.082364 \\
\hline 4 & 6 & 0 & -3.126892 & -1.895487 & -0.019585 \\
\hline 5 & 6 & 0 & -4.118040 & -1.069543 & 0.482919 \\
\hline 6 & 6 & 0 & -0.371965 & 0.376168 & 0. \\
\hline 7 & 6 & 0 & 2 & 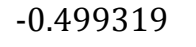 & 0. \\
\hline 8 & 16 & 0 & -0.223068 & $3-1.964951$ & -0.364365 \\
\hline 9 & 16 & 0 & -3.776163 & -3.379709 & -0.630867 \\
\hline 10 & 6 & 0 & -5.393471 & -2.877894 & -0.204310 \\
\hline 11 & 6 & 0 & -5.416918 & -1.635844 & 0.375921 \\
\hline 12 & 6 & 0 & 2.017272 & -0.339176 & 0.159298 \\
\hline 13 & 6 & 0 & 2.690876 & 0.487701 & 1.078434 \\
\hline 14 & 6 & 0 & 4.066870 & 0.665160 & 1.007695 \\
\hline 15 & 6 & 0 & 4.839951 & 0.017399 & 0.027874 \\
\hline 16 & 6 & 0 & 4.166830 & -0.815608 & -0.881962 \\
\hline 17 & 6 & 0 & 2.788798 & -0.987901 & -0.821866 \\
\hline 18 & 6 & 0 & 6.309951 & 0.203686 & -0.040076 \\
\hline 19 & 6 & 0 & 55 & -0.8 & 143 \\
\hline 20 & 6 & 0 & 8.53 & -0.686299 & 5202 \\
\hline 21 & 6 & 0 & 9.10 & 0.557230 & 849 \\
\hline 22 & 6 & 0 & 8.276153 & 1.624439 & 0.182763 \\
\hline 23 & 6 & 0 & 6.894768 & 1.449740 & 0.245463 \\
\hline 24 & 8 & 0 & -3.629671 & 0.843601 & 2.564465 \\
\hline 25 & 6 & 0 & -3.814373 & 1.815823 & -0.023969 \\
\hline 26 & 6 & 0 & -3.650106 & 1.706585 & -1.412661 \\
\hline 27 & 6 & 0 & -4.004120 & 2.768818 & -2.242241 \\
\hline 28 & 6 & 0 & -4.523448 & 3.944336 & -1.691501 \\
\hline 29 & 6 & 0 & -4.688283 & 4.056366 & -0.310560 \\
\hline 30 & 6 & 0 & -4.334717 & 2.994388 & 0.524629 \\
\hline 31 & 1 & 0 & -0.104464 & 1.345586 & 1.157688 \\
\hline 32 & 1 & 0 & 29 & -3 & 76 \\
\hline 33 & 1 & 0 & -6.32 & $7-1.1$ & 558 \\
\hline 34 & 1 & 0 & 2.13 & 0.9 & 33 \\
\hline 35 & 1 & 0 & 4.558125 & 1.286292 & 1.751206 \\
\hline 36 & 1 & 0 & 4.727098 & -1.307495 & -1.671980 \\
\hline 37 & 1 & 0 & 2.300643 & -1.610940 & -1.566871 \\
\hline 38 & 1 & 0 & 6.731502 & -1.840458 & -0.588813 \\
\hline 39 & 1 & 0 & 9.175561 & -1.526499 & -0.720716 \\
\hline 40 & 1 & 0 & 10.182090 & $\begin{array}{ll}0 & 0.693474\end{array}$ & -0.216772 \\
\hline 41 & 1 & 0 & 8.704994 & 2.599239 & 0.399958 \\
\hline 42 & 1 & 0 & 6.258078 & 2.294874 & 0.492159 \\
\hline 43 & 1 & 0 & -3.245878 & 30.794999 & -1.845222 \\
\hline 44 & 1 & 0 & -3.875121 & 2.681509 & -3.317671 \\
\hline 45 & 1 & 0 & -4.798302 & 4.771359 & -2.340785 \\
\hline 46 & 1 & 0 & -5.091623 & 4.969591 & 0.118719 \\
\hline 47 & 1 & 0 & -4.455657 & 3.065532 & 1.601532 \\
\hline
\end{tabular}


Table S3. Cartesian coordinates for minimized structure of compound 5.

\begin{tabular}{|c|c|c|c|c|c|}
\hline \multirow{2}{*}{$\begin{array}{l}\text { Center } \\
\text { Number }\end{array}$} & \multicolumn{3}{|c|}{ Atomic Atomic } & \multicolumn{2}{|c|}{ Coordinates (Angstroms } \\
\hline & & Number & Type & $\mathrm{X}$ & 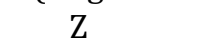 \\
\hline 1 & 15 & 0 & 0.000450 & 2.358359 & -1.166522 \\
\hline 2 & 6 & 0 & -1.303326 & 1.139911 & -0.745424 \\
\hline 3 & 6 & 0 & -0.722822 & -0.048792 & -0.332926 \\
\hline 4 & 6 & 0 & 0.725888 & -0.046496 & -0.327892 \\
\hline 5 & 6 & 0 & 1.305489 & 1.144276 & -0.735701 \\
\hline 6 & 6 & 0 & -2.716754 & 1.098047 & -0.737774 \\
\hline 7 & 6 & 0 & -3.225415 & -0.118696 & -0.322624 \\
\hline 8 & 16 & 0 & -1.913034 & -1.240640 & 0.060720 \\
\hline 9 & 16 & 0 & 1.917220 & -1.235815 & 0.070381 \\
\hline 10 & 6 & 0 & 3.228250 & -0.118584 & -0.330127 \\
\hline 11 & 6 & 0 & 2.718989 & 1.100853 & -0.736426 \\
\hline 12 & 6 & 0 & -4.625575 & -0.525845 & -0.195775 \\
\hline 13 & 6 & 0 & 4.628624 & -0.525548 & -0.204827 \\
\hline 14 & 6 & 0 & -5.623802 & 0.106846 & -0.961377 \\
\hline 15 & 6 & 0 & -6.959099 & -0.255919 & -0.838546 \\
\hline 16 & 6 & 0 & -7.363824 & -1.275544 & 0.041269 \\
\hline 17 & 6 & 0 & -6.36 & 2484 & $0.7 \mathrm{C}$ \\
\hline 18 & 6 & 0 & -5.0 & 5251 & 0.68 \\
\hline 19 & 6 & 0 & 31 & 219 & -0 \\
\hline 20 & 6 & 0 & 77 & 80 & -0 . \\
\hline 21 & 6 & 0 & 7.3 & 19 & 63 \\
\hline 22 & 6 & 0 & 6.967052 & 0.068628 & 0.12 \\
\hline 23 & 6 & 0 & 5.631864 & 0.436543 & 0.01 \\
\hline 24 & 6 & 0 & -8.789601 & -1.665481 & 0.163645 \\
\hline 25 & 6 & 0 & 8.792059 & -1.666339 & 0.152833 \\
\hline 26 & 6 & 0 & -9.158082 & -3.007865 & 0.358986 \\
\hline 27 & 6 & 0 & -10.498057 & $7 \quad-3.374208$ & $8 \quad 0.473148$ \\
\hline 28 & 6 & 0 & -11.501164 & $4 \quad-2.406383$ & $\begin{array}{ll}3 & 0.393829\end{array}$ \\
\hline 29 & 6 & 0 & -11.151144 & $4 \quad-1.068848$ & $8 \quad 0.199721$ \\
\hline 30 & 6 & 0 & -9.811026 & -0.702822 & 0.086382 \\
\hline 31 & 6 & 0 & 9.812435 & -0.850789 & -0.366567 \\
\hline 32 & 6 & 0 & 11.152287 & $7-1.216402$ & $2-0.249001$ \\
\hline 33 & 6 & 0 & 90 & -2.4 & 734 \\
\hline 34 & 6 & 0 & 10.5 & -3.2 & $1 \quad 0.9$ \\
\hline 35 & 6 & 0 & 9.161562 & -2.86 & 0.793365 \\
\hline 36 & 8 & 0 & 0.003400 & 127 & -2.541843 \\
\hline 37 & 6 & 0 & -0.004994 & 3.628752 & 0.146267 \\
\hline 38 & 6 & 0 & -0.009303 & 3.310836 & 1.512319 \\
\hline 39 & 6 & 0 & -0.013159 & 4.327703 & 2.465131 \\
\hline 40 & 6 & 0 & -0.012719 & 5.666080 & 2.060653 \\
\hline 41 & 6 & 0 & -0.008407 & 5.985962 & 0.702560 \\
\hline 42 & 6 & 0 & -0.004538 & 4.970078 & -0.255688 \\
\hline 43 & 1 & 0 & -3.350365 & 1.936045 & -1.005078 \\
\hline 44 & 1 & 0 & 3.351646 & 1.920634 & -1.057275 \\
\hline 45 & 1 & 0 & -5.343843 & 0.868486 & -1.682993 \\
\hline 46 & 1 & 0 & -7.698812 & 0.233068 & -1.465997 \\
\hline 47 & 1 & 0 & -6.643279 & -2.683093 & 1.510209 \\
\hline 48 & 1 & 0 & -4.292044 & -2.036894 & 1.315686 \\
\hline
\end{tabular}

$-\mathrm{S} 15-$ 


$\begin{array}{cccccc}49 & 1 & 0 & 4.285128 & -2.638870 & -0.510599 \\ 50 & 1 & 0 & 6.637422 & -3.280324 & -0.300460 \\ 51 & 1 & 0 & 7.710853 & 0.833672 & 0.330342 \\ 52 & 1 & 0 & 5.355576 & 1.479566 & 0.142561 \\ 53 & 1 & 0 & -8.388049 & -3.773526 & 0.393265 \\ 54 & 1 & 0 & -10.759151 & -4.419656 & 0.615028 \\ 55 & 1 & 0 & -12.545865 & -2.691805 & 0.482175 \\ 56 & 1 & 0 & -11.923150 & -0.305777 & 0.144915 \\ 57 & 1 & 0 & -9.549481 & 0.344448 & -0.036994 \\ 58 & 1 & 0 & 9.550297 & 0.063886 & -0.891017 \\ 59 & 1 & 0 & 11.923251 & -0.573722 & -0.665927 \\ 60 & 1 & 0 & 12.547652 & -2.692623 & 0.481006 \\ 61 & 1 & 0 & 10.763097 & -4.153588 & 1.416859 \\ 62 & 1 & 0 & 8.392831 & -3.495050 & 1.227690 \\ 63 & 1 & 0 & -0.009746 & 2.272016 & 1.832308 \\ 64 & 1 & 0 & -0.016727 & 4.077852 & 3.522681 \\ 65 & 1 & 0 & -0.015895 & 6.457263 & 2.805785 \\ 66 & 1 & 0 & -0.008196 & 7.025774 & 0.386799 \\ 67 & 1 & 0 & -0.001237 & 5.201967 & -1.316597 \\ -----------------------------------------------------\end{array}$


Table S4. Cartesian coordinates for minimized structure of compound 6.

\begin{tabular}{|c|c|c|c|c|c|}
\hline \multirow{2}{*}{$\begin{array}{l}\text { Center } \\
\text { Number }\end{array}$} & \multicolumn{2}{|c|}{ Atomic } & Atomic & \multicolumn{2}{|c|}{ Coordinates (Angstrom } \\
\hline & & Number & Type & $\mathrm{X}$ & -2 \\
\hline 1 & 15 & 0 & -0.074675 & -0.942890 & 2.16286 \\
\hline 2 & 6 & 0 & -1.393987 & -0.876705 & 0.935579 \\
\hline 3 & 6 & 0 & -0.806831 & -0.840288 & -0.324386 \\
\hline 4 & 6 & 0 & 0.638955 & -0.889823 & -0.327862 \\
\hline 5 & 6 & 0 & 1.230732 & -0.974363 & 0.929369 \\
\hline 6 & 6 & 0 & -2.809156 & -0.866104 & 0.910583 \\
\hline 7 & 6 & 0 & -3.316503 & -0.809767 & -0.375531 \\
\hline 8 & 16 & 0 & -2.004439 & -0.763596 & -1.561974 \\
\hline 9 & 16 & 0 & 1.831919 & -0.858045 & -1.570048 \\
\hline 10 & 6 & 0 & 3.147505 & -0.937296 & -0.390831 \\
\hline 11 & 6 & 0 & 2.646722 & -0.996586 & 0.897019 \\
\hline 12 & 6 & 0 & -4.714537 & -0.784663 & -0.805108 \\
\hline 13 & 6 & 0 & 4.542815 & -0.943738 & -0.829632 \\
\hline 14 & 6 & 0 & -5.719511 & -0.321200 & 0.065544 \\
\hline 15 & 6 & 0 & -7.051767 & -0.306049 & -0.325265 \\
\hline 16 & 6 & 0 & -7.445622 & -0.741571 & -1.604138 \\
\hline 17 & 6 & 0 & -6.437802 & -1.196923 & -2.473015 \\
\hline 18 & 6 & 0 & -5.1 & 1979 & -2.0 \\
\hline 19 & 6 & 0 & 4.9 & 583 & -2 \\
\hline 20 & 6 & 0 & 6.2 & 03 & 99 \\
\hline 21 & 6 & 0 & 7.2 & 13 & -1 \\
\hline 22 & 6 & 0 & 6.86 & 0342 & -0.4 \\
\hline 23 & 6 & 0 & 5.53 & 0605 & -0.0 \\
\hline 24 & 6 & 0 & -8.867908 & -0.719606 & -2.019653 \\
\hline 25 & 6 & 0 & 8.685496 & -0.963899 & -2.073623 \\
\hline 26 & 6 & 0 & -9.227523 & -0.403128 & -3.341119 \\
\hline 27 & 6 & 0 & -10.565128 & $8-0.382305$ & $5-3.731168$ \\
\hline 28 & 6 & 0 & -11.570400 & $0-0.680559$ & $9-2.809244$ \\
\hline 29 & 6 & 0 & -11.227901 & $1 \quad-0.998008$ & $8-1.493440$ \\
\hline 30 & 6 & 0 & -9.890686 & -1.015703 & -1.101934 \\
\hline 31 & 6 & 0 & 9.504809 & 577 & -1.833541 \\
\hline 32 & 6 & 0 & 10 & $7-2.0$ & 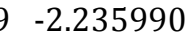 \\
\hline 33 & 6 & 0 & 11 & $5-0$ & $7 \quad-2$ \\
\hline 34 & 6 & 0 & 10.5 & 0.1 & -3 \\
\hline 35 & 6 & 0 & 916 & 0.14 & -2.7 \\
\hline 36 & 6 & 0 & -0.102292 & -2.4 & 775 \\
\hline 37 & 6 & 0 & 0.6 & -3.5 & 2.8 \\
\hline 38 & 6 & 0 & 0.626966 & -4.680840 & 3.660318 \\
\hline 39 & 6 & 0 & -0.187442 & -4.713706 & 4.793742 \\
\hline 40 & 6 & 0 & -0.960786 & -3.601886 & 5.137435 \\
\hline 41 & 6 & 0 & -0.921710 & -2.453628 & 4.349627 \\
\hline 42 & 1 & 0 & -3.444073 & -0.938541 & 1.785654 \\
\hline 43 & 1 & 0 & 3.286177 & -1.022763 & 1.771324 \\
\hline 44 & 1 & 0 & -5.451211 & 0.059986 & 1.046873 \\
\hline 45 & 1 & 0 & -7.799398 & 0.084504 & 0.358469 \\
\hline 46 & 1 & 0 & -6.708294 & -1.571307 & -3.455418 \\
\hline 47 & 1 & 0 & -4.363230 & -1.619511 & -2.772691 \\
\hline 48 & 1 & 0 & 4.209186 & 0.179284 & -2.651204 \\
\hline 49 & 1 & 0 & 6.546268 & 0.123505 & -3.371556 \\
\hline 50 & 1 & 0 & 7.604998 & -2.042354 & 0.187751 \\
\hline
\end{tabular}




$\begin{array}{lllccc}51 & 1 & 0 & 5.252406 & -2.075208 & 0.872669 \\ 52 & 1 & 0 & -8.456134 & -0.142337 & -4.060323 \\ 53 & 1 & 0 & -10.822870 & -0.125360 & -4.754785 \\ 54 & 1 & 0 & -12.612906 & -0.666025 & -3.113961 \\ 55 & 1 & 0 & -12.003016 & -1.240754 & -0.771912 \\ 56 & 1 & 0 & -9.634649 & -1.292229 & -0.082865 \\ 57 & 1 & 0 & 9.085449 & -2.961893 & -1.356244 \\ 58 & 1 & 0 & 11.452412 & -2.964127 & -2.051147 \\ 59 & 1 & 0 & 12.422809 & -0.980171 & -3.195985 \\ 60 & 1 & 0 & 10.997896 & 1.013936 & -3.622076 \\ 61 & 1 & 0 & 8.640000 & 1.032466 & -2.898214 \\ 62 & 1 & 0 & 1.309190 & -3.514086 & 1.984301 \\ 63 & 1 & 0 & 1.228847 & -5.544554 & 3.394966 \\ 64 & 1 & 0 & -0.219577 & -5.605895 & 5.411898 \\ 65 & 1 & 0 & -1.592575 & -3.627608 & 6.019948 \\ 66 & 1 & 0 & -1.527877 & -1.596780 & 4.630237 \\ 67 & 6 & 0 & -0.032323 & 0.572308 & 3.230765 \\ 68 & 1 & 0 & -0.965657 & 0.570869 & 3.806249 \\ 69 & 1 & 0 & 0.785944 & 0.409646 & 3.941283 \\ 70 & 6 & 0 & 0.147215 & 1.854897 & 2.453358 \\ 71 & 6 & 0 & -0.965733 & 2.516570 & 1.912122 \\ 72 & 6 & 0 & 1.421458 & 2.377677 & 2.273739 \\ 73 & 6 & 0 & -0.780004 & 3.692940 & 1.184117 \\ 74 & 1 & 0 & -1.960054 & 2.109203 & 2.049535 \\ 75 & 6 & 0 & 1.620815 & 3.570576 & 1.556839 \\ 76 & 1 & 0 & 2.299883 & 1.895947 & 2.691184 \\ 77 & 6 & 0 & 0.515161 & 4.241409 & 1.003072 \\ 78 & 8 & 0 & 2.922438 & 3.930772 & 1.428936 \\ 79 & 8 & 0 & 0.703212 & 5.339830 & 0.207780 \\ 80 & 8 & 0 & -1.785770 & 4.382194 & 0.581298 \\ 81 & 6 & 0 & 3.320038 & 5.289173 & 1.212270 \\ 82 & 1 & 0 & 4.393952 & 5.301256 & 1.409656 \\ 83 & 1 & 0 & 3.122775 & 5.608464 & 0.188080 \\ 84 & 1 & 0 & 2.818012 & 5.964762 & 1.914120 \\ 85 & 6 & 0 & 0.156305 & 6.585916 & 0.666925 \\ 86 & 1 & 0 & 0.454002 & 7.329133 & -0.074974 \\ 87 & 1 & 0 & -0.932743 & 6.541962 & 0.730400 \\ 88 & 1 & 0 & 0.575536 & 6.857194 & 1.644212 \\ 89 & 6 & 0 & -3.110154 & 3.871071 & 0.660895 \\ 90 & 1 & 0 & -3.728043 & 4.560968 & 0.084799 \\ 91 & 1 & 0 & -3.177878 & 2.867755 & 0.221690 \\ 92 & 1 & 0 & -3.468211 & 3.842824 & 1.698117 \\ ------------------------------------------------------\end{array}$

-S18- 
Table S5. Cartesian coordinates for minimized structure of compound 3-ox.

\begin{tabular}{|c|c|c|c|c|c|}
\hline \multirow{2}{*}{$\begin{array}{l}\text { Center } \\
\text { Number }\end{array}$} & \multicolumn{2}{|c|}{ Atomic } & Atomic & \multicolumn{2}{|c|}{ Coordinates (Angstrom } \\
\hline & & Number & Type & Y & 8 \\
\hline 1 & 15 & 0 & -0.000001 & 1.592541 & -1.123858 \\
\hline 2 & 6 & 0 & -1.295552 & 0.385462 & -0.598946 \\
\hline 3 & 6 & 0 & -0.705660 & -0.806018 & -0.112813 \\
\hline 4 & 6 & 0 & 0.705652 & -0.806021 & -0.112818 \\
\hline 5 & 6 & 0 & 1.295548 & 0.385455 & -0.598957 \\
\hline 6 & 6 & 0 & -2.681396 & 0.333536 & -0.617293 \\
\hline 7 & 6 & 0 & -3.201856 & -0.885342 & -0.140597 \\
\hline 8 & 16 & 0 & -1.896593 & -1.984509 & 0.327102 \\
\hline 9 & 16 & 0 & 1.896582 & -1.984519 & 0.327089 \\
\hline 10 & 6 & 0 & 3.201848 & -0.885352 & -0.140602 \\
\hline 11 & 6 & 0 & 2.681391 & 0.333523 & -0.617307 \\
\hline 12 & 6 & 0 & -4.589075 & -1.273882 & $2-0.017406$ \\
\hline 13 & 6 & 0 & 4.589066 & -1.273894 & -0.017409 \\
\hline 14 & 6 & 0 & -5.602633 & -0.488247 & $7 \quad-0.619284$ \\
\hline 15 & 6 & 0 & -6.936797 & $7-0.852936$ & $5-0.508307$ \\
\hline 16 & 6 & 0 & -7.295525 & -2.003760 & 0.202606 \\
\hline 17 & 6 & 0 & -6.307177 & $7-2.792735$ & 0.802883 \\
\hline 18 & 6 & 0 & -4.971094 & -2.435417 & $7 \quad 0.695078$ \\
\hline 19 & 6 & 0 & $4.9 ?$ & -2.4 & 01 \\
\hline 20 & 6 & 0 & 6.30 & -2.792733 & 907 \\
\hline 21 & 6 & 0 & 7.295515 & -2.003775 & 0.202607 \\
\hline 22 & 6 & 0 & 6.936787 & -0.852966 & -0.508331 \\
\hline 23 & 6 & 0 & 5.602622 & -0.488276 & -0.619310 \\
\hline 24 & 8 & 0 & -0.000009 & 1.987652 & -2.564027 \\
\hline 25 & 6 & 0 & 0.000010 & 2.969139 & 0.048524 \\
\hline 26 & 6 & 0 & 0.000019 & 2.780423 & 1.440203 \\
\hline 27 & 6 & 0 & 0.000028 & 3.885813 & 2.287210 \\
\hline 28 & 6 & 0 & 0.000028 & 5.177370 & 1.750400 \\
\hline 29 & 6 & 0 & 0.000020 & 5.366239 & 0.367457 \\
\hline 30 & 6 & 0 & 0.000011 & 4.264403 & -0.489089 \\
\hline 31 & 1 & 0 & -3.312881 & 1.149254 & -0.946903 \\
\hline 32 & 1 & 0 & 79 & 1.12 & -0.946917 \\
\hline 33 & 1 & 0 & -5.340104 & 0.394548 & -1.191991 \\
\hline 34 & 1 & 0 & -7.701249 & -0.244215 & -0.980824 \\
\hline 35 & 1 & 0 & -8.340603 & -2.285632 & 0.287646 \\
\hline 36 & 1 & 0 & -6.583592 & -3.683673 & $\begin{array}{ll}3 & 1.357808\end{array}$ \\
\hline 37 & 1 & 0 & -4.218515 & -3.049700 & 1.181751 \\
\hline 38 & 1 & 0 & 4.218507 & -3.049682 & 1.181792 \\
\hline 39 & 1 & 0 & 6.583583 & -3.683659 & 1.357852 \\
\hline 40 & 1 & 0 & 8.340592 & -2.285647 & 0.287648 \\
\hline 41 & 1 & 0 & 7.701237 & -0.244259 & -0.980867 \\
\hline 42 & 1 & 0 & 5.340092 & 0.394505 & -1.192038 \\
\hline 43 & 1 & 0 & 0.000018 & 1.779160 & 1.864334 \\
\hline 44 & 1 & 0 & 0.000034 & 3.743127 & 3.363763 \\
\hline 45 & 1 & 0 & 0.000036 & 6.037107 & 2.414193 \\
\hline 46 & 1 & 0 & 0.000021 & 6.370092 & -0.046495 \\
\hline 47 & 1 & 0 & 0.000005 & 4.396968 & -1.566770 \\
\hline
\end{tabular}


Table S6. Cartesian coordinates for minimized structure of compound 4-ox.

\begin{tabular}{|c|c|c|c|c|c|}
\hline \multirow{2}{*}{$\begin{array}{l}\text { Center } \\
\text { Number }\end{array}$} & \multicolumn{2}{|r|}{ tomic } & Atomic & \multicolumn{2}{|c|}{ Coordinates (Angstrom } \\
\hline & & Number & Type & $X$ & Z \\
\hline 1 & 15 & 0 & -3.356230 & 0.460275 & 1.114326 \\
\hline 2 & 6 & 0 & -1.674701 & -0.096140 & 0.580480 \\
\hline 3 & 6 & 0 & -1.740687 & -1.382682 & -0.005698 \\
\hline 4 & 6 & 0 & -3.048616 & -1.929822 & -0.083216 \\
\hline 5 & 6 & 0 & -4.059892 & -1.105125 & 0.442799 \\
\hline 6 & 6 & 0 & -0.380791 & 0.385196 & 0.67 \\
\hline 7 & 6 & 0 & 9 & -0.502916 & 95 \\
\hline 8 & 16 & 0 & -0.181372 & -1.979451 & 49174 \\
\hline 9 & 16 & 0 & -3.679787 & -3.437862 & -0.668209 \\
\hline 10 & 6 & 0 & -5.281681 & -2.955860 & -0.203076 \\
\hline 11 & 6 & 0 & -5.331450 & -1.699246 & 0.373326 \\
\hline 12 & 6 & 0 & 2.010588 & -0.325097 & 0.107021 \\
\hline 13 & 6 & 0 & 2.617022 & 0.854690 & 0.621750 \\
\hline 14 & 6 & 0 & 3.982387 & 1.033276 & 0.571570 \\
\hline 15 & 6 & 0 & 4.840125 & 0.051434 & 0.014443 \\
\hline 16 & 6 & 0 & 4.234977 & -1.125539 & -0.495291 \\
\hline 17 & 6 & 0 & 2.869993 & -1.307929 & -0.454898 \\
\hline 18 & 6 & 0 & 6.293017 & 0.244094 & -0.032767 \\
\hline 19 & 6 & 0 & 0 & 066 & 33 \\
\hline 20 & 6 & 0 & 3 & -0.6 & \\
\hline 21 & 6 & 0 & 9.0 & 0.6 & 90 \\
\hline 22 & 6 & 0 & 8.227399 & 1.720571 & 9541 \\
\hline 23 & 6 & 0 & 6.850587 & 1.540454 & -0.097339 \\
\hline 24 & 8 & 0 & -3.529272 & 0.715495 & 2.575697 \\
\hline 25 & 6 & 0 & -3.872836 & 1.817601 & 0.035926 \\
\hline 26 & 6 & 0 & -3.805714 & 1.743271 & -1.364879 \\
\hline 27 & 6 & 0 & -4.222366 & 2.826170 & -2.135092 \\
\hline 28 & 6 & 0 & -4.706795 & 3.981205 & -1.512655 \\
\hline 29 & 6 & 0 & -4.775806 & 4.055657 & -0.120549 \\
\hline 30 & 6 & 0 & -4.359573 & 2.975690 & 0.659238 \\
\hline 31 & 1 & 0 & -0.126440 & 1.345710 & 1.107960 \\
\hline 32 & 1 & 0 & -6 & -3.6 & -0.37 \\
\hline 33 & 1 & 0 & & -1.2 & 998 \\
\hline 34 & 1 & 0 & 3 & 29 & 1.0 \\
\hline 35 & 1 & 0 & 4.408398 & 1.931695 & 1.004251 \\
\hline 36 & 1 & 0 & 4.852329 & -1.886190 & -0.960111 \\
\hline 37 & 1 & 0 & 2.453346 & -2.219379 & -0.874151 \\
\hline 38 & 1 & 0 & 6.774343 & -1.868429 & 0.071796 \\
\hline 39 & 1 & 0 & 9.208613 & -1.538423 & -0.018775 \\
\hline 40 & 1 & 0 & 10.157798 & $\begin{array}{ll}3 & 0.756085\end{array}$ & -0.157879 \\
\hline 41 & 1 & 0 & 8.637485 & 2.723618 & -0.216551 \\
\hline 42 & 1 & 0 & 6.200535 & 2.408028 & -0.149351 \\
\hline 43 & 1 & 0 & -3.435052 & 0.846631 & -1.855790 \\
\hline 44 & 1 & 0 & -4.173176 & 2.770758 & -3.218555 \\
\hline 45 & 1 & 0 & -5.032917 & 4.822947 & -2.116730 \\
\hline 46 & 1 & 0 & 5.155125 & 4.952390 & 0.360173 \\
\hline 47 & 1 & 0 & -4.411096 & 3.018546 & 1.742948 \\
\hline
\end{tabular}


Table S7. Cartesian coordinates for minimized structure of compound 5-ox.

\begin{tabular}{|c|c|c|c|c|c|}
\hline \multirow{2}{*}{$\begin{array}{l}\text { Center } \\
\text { Number }\end{array}$} & \multicolumn{3}{|c|}{ Atomic Atomic } & \multicolumn{2}{|c|}{ Coordinates (Angstroms } \\
\hline & & Number & Type & $\mathrm{X}$ & 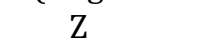 \\
\hline 1 & 15 & 0 & 0.000450 & 2.358359 & -1.166522 \\
\hline 2 & 6 & 0 & -1.303326 & 1.139911 & -0.745424 \\
\hline 3 & 6 & 0 & -0.722822 & -0.048792 & -0.332926 \\
\hline 4 & 6 & 0 & 0.725888 & -0.046496 & -0.327892 \\
\hline 5 & 6 & 0 & 1.305489 & 1.144276 & -0.735701 \\
\hline 6 & 6 & 0 & -2.716754 & 1.098047 & -0.737774 \\
\hline 7 & 6 & 0 & -3.225416 & -0.118696 & -0.322624 \\
\hline 8 & 16 & 0 & -1.913035 & -1.240640 & 0.060720 \\
\hline 9 & 16 & 0 & 1.917221 & -1.235815 & 0.070381 \\
\hline 10 & 6 & 0 & 3.228250 & -0.118584 & -0.330127 \\
\hline 11 & 6 & 0 & 2.718990 & 1.100854 & -0.736426 \\
\hline 12 & 6 & 0 & -4.625575 & -0.525845 & -0.195775 \\
\hline 13 & 6 & 0 & 4.628625 & -0.525548 & -0.204828 \\
\hline 14 & 6 & 0 & -5.623802 & 0.106846 & -0.961377 \\
\hline 15 & 6 & 0 & -6.959099 & -0.255919 & -0.838546 \\
\hline 16 & 6 & 0 & -7.363824 & -1.275544 & 0.041269 \\
\hline 17 & 6 & 0 & -6.36 & 2484 & $0.7 \mathrm{C}$ \\
\hline 18 & 6 & 0 & -5.0 & 5251 & 0.68 \\
\hline 19 & 6 & 0 & 32 & 219 & -0 \\
\hline 20 & 6 & 0 & 8 & 81 & -0 . \\
\hline 21 & 6 & 0 & 7.3 & 19 & 63 \\
\hline 22 & 6 & 0 & 6.967052 & 0.068628 & 0.12 \\
\hline 23 & 6 & 0 & 5.631865 & 0.436543 & 0.01 \\
\hline 24 & 6 & 0 & -8.789602 & -1.665481 & 0.163645 \\
\hline 25 & 6 & 0 & 8.792060 & -1.666339 & 0.152833 \\
\hline 26 & 6 & 0 & -9.158082 & -3.007865 & 0.358986 \\
\hline 27 & 6 & 0 & -10.498057 & $7 \quad-3.374208$ & $8 \quad 0.473148$ \\
\hline 28 & 6 & 0 & -11.501165 & $5-2.406383$ & $\begin{array}{ll}3 & 0.393829\end{array}$ \\
\hline 29 & 6 & 0 & -11.151145 & $5-1.068848$ & $8 \quad 0.199721$ \\
\hline 30 & 6 & 0 & -9.811027 & -0.702822 & 0.086382 \\
\hline 31 & 6 & 0 & 9.812436 & -0.850789 & -0.366567 \\
\hline 32 & 6 & 0 & 11.152287 & $7-1.216402$ & $2-0.249001$ \\
\hline 33 & 6 & 0 & 91 & L -2.4 & 734 \\
\hline 34 & 6 & 0 & 10.5 & -3.2 & $1 \quad 0.9$ \\
\hline 35 & 6 & 0 & 9.161562 & -2.86 & 0.793365 \\
\hline 36 & 8 & 0 & 0.003400 & 127 & -2.541843 \\
\hline 37 & 6 & 0 & -0.004994 & 3.628753 & 0.146267 \\
\hline 38 & 6 & 0 & -0.009303 & 3.310836 & 1.512319 \\
\hline 39 & 6 & 0 & -0.013159 & 4.327704 & 2.465131 \\
\hline 40 & 6 & 0 & -0.012719 & 5.666080 & 2.060653 \\
\hline 41 & 6 & 0 & -0.008407 & 5.985962 & 0.702560 \\
\hline 42 & 6 & 0 & -0.004538 & 4.970079 & -0.255688 \\
\hline 43 & 1 & 0 & -3.350365 & 1.936046 & -1.005078 \\
\hline 44 & 1 & 0 & 3.351646 & 1.920634 & -1.057275 \\
\hline 45 & 1 & 0 & -5.343843 & 0.868486 & -1.682993 \\
\hline 46 & 1 & 0 & -7.698812 & 0.233068 & -1.465997 \\
\hline 47 & 1 & 0 & -6.643279 & -2.683093 & 1.510209 \\
\hline 48 & 1 & 0 & -4.292044 & -2.036894 & 1.315686 \\
\hline
\end{tabular}




\begin{tabular}{|c|c|c|c|c|c|}
\hline 49 & 1 & 0 & 4.285128 & -2.638870 & -0.510599 \\
\hline 50 & 1 & 0 & 6.637423 & -3.280325 & -0.300460 \\
\hline 51 & 1 & 0 & 7.710853 & 0.833672 & 0.330342 \\
\hline 52 & 1 & 0 & 5.355576 & 1.479566 & 0.142561 \\
\hline 53 & 1 & 0 & -8.388050 & -3.773527 & 0.393265 \\
\hline 54 & 1 & 0 & -10.759152 & -4.419656 & 0.615028 \\
\hline 55 & 1 & 0 & -12.545866 & -2.691806 & 0.482175 \\
\hline 56 & 1 & 0 & -11.923151 & -0.305777 & 0.144915 \\
\hline 57 & 1 & 0 & -9.549482 & 0.344448 & -0.036994 \\
\hline 58 & 1 & 0 & 9.550298 & 0.063886 & -0.891017 \\
\hline 59 & 1 & 0 & 11.923251 & -0.573722 & -0.665927 \\
\hline 60 & 1 & 0 & 12.547653 & -2.692623 & 0.481006 \\
\hline 61 & 1 & 0 & 10.763097 & -4.153588 & 1.416859 \\
\hline 62 & 1 & 0 & 8.392832 & -3.495050 & 1.227690 \\
\hline 63 & 1 & 0 & -0.009746 & 2.272017 & 1.832308 \\
\hline 64 & 1 & 0 & -0.016727 & 4.077852 & 3.522681 \\
\hline 65 & 1 & 0 & -0.015895 & 6.457263 & 2.805785 \\
\hline 66 & 1 & 0 & -0.008196 & 7.025775 & 0.386799 \\
\hline & 1 & 0 & -0.001237 & 5.201967 & -1.316597 \\
\hline
\end{tabular}


Table S8. Cartesian coordinates for minimized structure of compound 5-ox2.

\begin{tabular}{|c|c|c|c|c|c|}
\hline \multirow{2}{*}{$\begin{array}{l}\text { Center } \\
\text { Number }\end{array}$} & \multicolumn{2}{|c|}{ Ator } & Atomic & \multicolumn{2}{|c|}{ Coordinates (Angstroms } \\
\hline & & Number & Type & $X$ & Z \\
\hline 1 & 15 & 0 & -0.000698 & 2.375082 & -1.07033 \\
\hline 2 & 6 & 0 & -1.293374 & 1.145477 & -0.568259 \\
\hline 3 & 6 & 0 & -0.696147 & -0.076853 & -0.119018 \\
\hline 4 & 6 & 0 & 0.696592 & -0.075908 & -0.118001 \\
\hline 5 & 6 & 0 & 1.292844 & 1.147469 & -0.565671 \\
\hline 6 & 6 & 0 & -2.663046 & 1.088654 & -0 . \\
\hline 7 & 6 & 0 & -3 & -0.1 & -0 \\
\hline 8 & 16 & 0 & -1.888291 & -1.278903 & 0.266841 \\
\hline 9 & 16 & 0 & 1.889870 & -1.275492 & 0.2 \\
\hline 10 & 6 & 0 & $3.1 \mathrm{c}$ & 2179 & -0.17 \\
\hline 11 & 6 & 0 & 2.662733 & 1.09 & -0.6 \\
\hline 12 & 6 & 0 & -4.558143 & -0.550780 & -0.115539 \\
\hline 13 & 6 & 0 & 4.559263 & -0.544331 & -0.108037 \\
\hline 14 & 6 & 0 & -5.594763 & 0.353040 & -0.505881 \\
\hline 15 & 6 & 0 & -6.916632 & -0.012699 & -0.443732 \\
\hline 16 & 6 & 0 & -7.315196 & -1.306324 & 0.000513 \\
\hline 17 & 6 & 0 & -6.279368 & -2.207600 & 0.386792 \\
\hline 18 & 6 & 0 & -4.956900 & -1.845329 & 6678 \\
\hline 19 & 6 & 0 & 8 & 731 & 12 \\
\hline 20 & 6 & 0 & 0 & 31 & \\
\hline 21 & 0 & 0 & & -1 & \\
\hline 22 & 6 & 0 & 6.9 & -0.0 & -0 . \\
\hline 23 & 6 & 0 & 5.59 & 88 & -0.5 \\
\hline 24 & 6 & 0 & -8.7 & -1.6 & 174 \\
\hline 25 & 6 & 0 & 8.7 & -1.6 & 80 \\
\hline 26 & 6 & 0 & -9.098486 & -3.059040 & 0.022080 \\
\hline 27 & 6 & 0 & -10.435410 & $0 \quad-3.422970$ & $\begin{array}{ll}0 & 0.062078\end{array}$ \\
\hline 28 & 6 & 0 & -11.428087 & $\begin{array}{ll}7 & -2.438739\end{array}$ & $\begin{array}{ll}9 & 0.159786\end{array}$ \\
\hline 29 & 6 & 0 & -11.073898 & $8 \quad-1.083681$ & $\begin{array}{ll}1 & 0.207088\end{array}$ \\
\hline 30 & 6 & 0 & -9.739753 & -0.712746 & 0.146351 \\
\hline 31 & 6 & 0 & 9.682910 & -1.048702 & -0.757911 \\
\hline 32 & 6 & 0 & 11.016185 & $5-1.424566$ & $5-0.707863$ \\
\hline 33 & 6 & 0 & 96 & $5-2.4$ & 05 \\
\hline 34 & 6 & 0 & & $3-3$. & 498 \\
\hline 35 & 6 & 0 & 76 & -2.7 & 86 \\
\hline 36 & 8 & 0 & 37 & 99 & -2.5 \\
\hline 37 & 6 & 0 & -0.0 & 80 & 0.1 \\
\hline 38 & 6 & 0 & -0.0 & 10 & 1.502401 \\
\hline 39 & 6 & 0 & -0.004197 & 4.623600 & 2.359866 \\
\hline 40 & 6 & 0 & -0.004631 & 5.920743 & 1.835058 \\
\hline 41 & 6 & 0 & -0.004010 & 6.125397 & 0.453831 \\
\hline 42 & 6 & 0 & -0.002908 & 5.033771 & -0.415167 \\
\hline 43 & 1 & 0 & -3.291492 & 1.907481 & -0.936815 \\
\hline 44 & 1 & 0 & 3.290658 & 1.913486 & -0.927663 \\
\hline 45 & 1 & 0 & -5.345521 & 1.341613 & -0.873698 \\
\hline 46 & 1 & 0 & -7.666776 & 0.692129 & -0.782185 \\
\hline 47 & 1 & 0 & 6.537079 & -3.18916 & 0.766310 \\
\hline 48 & 1 & 0 & -4.208155 & -2.562514 & 0.660675 \\
\hline
\end{tabular}




\begin{tabular}{|c|c|c|c|c|c|}
\hline 49 & 1 & 0 & 4.210645 & -2.555200 & 0.671044 \\
\hline 50 & 1 & 0 & 6.539243 & -3.186336 & 0.760769 \\
\hline 51 & 1 & 0 & 7.668370 & 0.707025 & -0.757531 \\
\hline 52 & 1 & 0 & 5.344853 & 1.346806 & -0.871289 \\
\hline 53 & 1 & 0 & -8.346538 & -3.833188 & -0.085362 \\
\hline 54 & 1 & 0 & -10.712324 & -4.471128 & 0.012398 \\
\hline 55 & 1 & 0 & -12.474283 & -2.726799 & 0.198969 \\
\hline 56 & 1 & 0 & -11.843204 & -0.323383 & 0.295767 \\
\hline 57 & 1 & 0 & -9.483169 & 0.338739 & 0.215177 \\
\hline 58 & 1 & 0 & 9.376250 & -0.294310 & -1.474240 \\
\hline 59 & 1 & 0 & 11.737842 & -0.945563 & -1.361729 \\
\hline 60 & 1 & 0 & 12.476487 & -2.713025 & 0.223990 \\
\hline 61 & 1 & 0 & 10.821247 & -3.824056 & 1.710319 \\
\hline 62 & 1 & 0 & 8.456676 & -3.179584 & 1.626734 \\
\hline 63 & 1 & 0 & -0.002705 & 2.521864 & 1.917933 \\
\hline 64 & 1 & 0 & -0.004641 & 4.471117 & 3.434861 \\
\hline 65 & 1 & 0 & -0.005404 & 6.773403 & 2.507604 \\
\hline 66 & 1 & 0 & -0.004330 & 7.133600 & 0.051261 \\
\hline & 1 & 0 & -0.002366 & 5.180072 & -1.491065 \\
\hline
\end{tabular}


Table S9. Cartesian coordinates for minimized structure of compound 3-red.

\begin{tabular}{|c|c|c|c|c|c|}
\hline \multirow{2}{*}{$\begin{array}{l}\text { Center } \\
\text { Number }\end{array}$} & \multicolumn{2}{|c|}{ Atomic } & Atomic & \multicolumn{2}{|c|}{ Coordinates (Angstrom } \\
\hline & & Number & Type & Y & 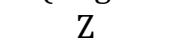 \\
\hline 1 & 15 & 0 & 0.000000 & 1.507585 & -1.199766 \\
\hline 2 & 6 & 0 & -1.296349 & 0.348497 & -0.684781 \\
\hline 3 & 6 & 0 & -0.702311 & -0.839709 & -0.180347 \\
\hline 4 & 6 & 0 & 0.702311 & -0.839709 & -0.180347 \\
\hline 5 & 6 & 0 & 1.296349 & 0.348497 & -0.684782 \\
\hline 6 & 6 & 0 & -2.693885 & 0.317138 & -0.667954 \\
\hline 7 & 6 & 0 & -3.246063 & -0.861956 & -0.167488 \\
\hline 8 & 16 & 0 & -1.929677 & $7-1.992546$ & 0.303240 \\
\hline 9 & 16 & 0 & 1.929676 & -1.992547 & 0.303239 \\
\hline 10 & 6 & 0 & 3.246063 & -0.861957 & -0.167489 \\
\hline 11 & 6 & 0 & 2.693885 & 0.317136 & -0.667956 \\
\hline 12 & 6 & 0 & -4.635009 & -1.224559 & $\begin{array}{l}9 \\
-0.008090\end{array}$ \\
\hline 13 & 6 & 0 & 4.635008 & -1.224559 & -0.008090 \\
\hline 14 & 6 & 0 & -5.679188 & -0.342050 & -0.391874 \\
\hline 15 & 6 & 0 & -7.015644 & -0.685472 & -0.237285 \\
\hline 16 & 6 & 0 & -7.384713 & - -1.922949 & 0.305527 \\
\hline 17 & 6 & 0 & -6.373736 & -2.808148 & $\begin{array}{l}3 \\
0.689169\end{array}$ \\
\hline 18 & 6 & 0 & -5.032327 & $7-2.471563$ & $\begin{array}{ll}3 & 0.537978\end{array}$ \\
\hline 19 & 6 & 0 & 5.0 & -2.4 & 7977 \\
\hline 20 & 6 & 0 & 6.37 & -2.8 & 9169 \\
\hline 21 & 6 & 0 & 7.384713 & -1.922950 & 0.305528 \\
\hline 22 & 6 & 0 & 7.015643 & -0.685473 & -0.237284 \\
\hline 23 & 6 & 0 & 5.679187 & -0.342050 & -0.391874 \\
\hline 24 & 8 & 0 & 0.000000 & 2.076400 & -2.597302 \\
\hline 25 & 6 & 0 & 0.000000 & 2.894401 & 0.016278 \\
\hline 26 & 6 & 0 & 0.000001 & 2.677284 & 1.401624 \\
\hline 27 & 6 & 0 & 0.000001 & 3.756791 & 2.284163 \\
\hline 28 & 6 & 0 & 0.000002 & 5.065446 & 1.791364 \\
\hline 29 & 6 & 0 & 0.000001 & 5.288813 & 0.413817 \\
\hline 30 & 6 & 0 & 0.000000 & 4.206854 & -0.470356 \\
\hline 31 & 1 & 0 & -3.309054 & 1.139673 & -1.018846 \\
\hline 32 & 1 & 0 & 3.30 & 1.139672 & -1.018849 \\
\hline 33 & 1 & 0 & -5.429589 & 0.625217 & -0.817964 \\
\hline 34 & 1 & 0 & -7.782846 & 0.022584 & -0.545641 \\
\hline 35 & 1 & 0 & -8.432190 & -2.187987 & $7 \quad 0.424518$ \\
\hline 36 & 1 & 0 & -6.631582 & -3.776887 & $7 \quad 1.113460$ \\
\hline 37 & 1 & 0 & -4.272098 & $\begin{array}{l}3.183750 \\
\end{array}$ & 0.847892 \\
\hline 38 & 1 & 0 & 4.272097 & -3.183751 & 0.847890 \\
\hline 39 & 1 & 0 & 6.631581 & -3.776888 & 1.113460 \\
\hline 40 & 1 & 0 & 8.432189 & -2.187988 & 0.424518 \\
\hline 41 & 1 & 0 & 7.782845 & 0.022583 & -0.545639 \\
\hline 42 & 1 & 0 & 5.429589 & 0.625217 & -0.817963 \\
\hline 43 & 1 & 0 & 0.000000 & 1.660637 & 1.785916 \\
\hline 44 & 1 & 0 & 0.000002 & 3.578938 & 3.357139 \\
\hline 45 & 1 & 0 & 0.000002 & 5.906774 & 2.481118 \\
\hline 46 & 1 & 0 & 0.000001 & 6.305604 & 0.026616 \\
\hline 47 & 1 & 0 & 0.000000 & 4.358626 & -1.545965 \\
\hline
\end{tabular}


Table S10. Cartesian coordinates for minimized structure of compound 4-red.

\begin{tabular}{|c|c|c|c|c|c|}
\hline \multirow{2}{*}{$\begin{array}{l}\text { Center } \\
\text { Number }\end{array}$} & \multicolumn{2}{|c|}{ Atomic } & Atomic & \multicolumn{2}{|c|}{ Coordinates (Angstrom } \\
\hline & & Numbe & Type & $\mathrm{X}$ & $\mathrm{Z}$ \\
\hline 1 & 15 & 0 & -3.371701 & 0.426678 & 1.147296 \\
\hline 2 & 6 & 0 & -1.714536 & -0.149922 & 0.673014 \\
\hline 3 & 6 & 0 & -1.812437 & -1.418405 & 0.043090 \\
\hline 4 & 6 & 0 & -3.130885 & -1.904959 & -0.073976 \\
\hline 5 & 6 & 0 & -4.135015 & -1.056197 & 0.451296 \\
\hline 6 & 6 & 0 & -0.405860 & 0.320448 & 0.775559 \\
\hline 7 & 6 & 0 & 0.567106 & -0.537545 & 0.246425 \\
\hline 8 & 16 & 0 & -0.227004 & 7352 & -0.40507 \\
\hline 9 & 16 & 0 & -3.834573 & -3.369765 & -0.734847 \\
\hline 10 & 6 & 0 & -5.460303 & -2.820556 & -0.303239 \\
\hline 11 & 6 & 0 & -5.438039 & -1.593172 & 0.309719 \\
\hline 12 & 6 & 0 & 1.988477 & -0.364138 & 0.180074 \\
\hline 13 & 6 & 0 & 2.632961 & 0.773902 & 0.743649 \\
\hline 14 & 6 & 0 & 4.001628 & 0.950500 & 0.671582 \\
\hline 15 & 6 & 0 & 4.851802 & 0.009205 & 0.043655 \\
\hline 16 & 6 & 0 & 4.218756 & -1.125199 & -0.509186 \\
\hline 17 & 6 & 0 & 2.847658 & -1.306906 & -0.449502 \\
\hline 18 & 6 & 0 & 6.308469 & 0.203210 & -0.030319 \\
\hline 19 & 6 & 0 & 41 & -0.891145 & -0.100277 \\
\hline 20 & 6 & 0 & 43 & -0.706534 & 858 \\
\hline 21 & 6 & 0 & & & \\
\hline 22 & 6 & 0 & 8.26 & 1.677156 & 342 \\
\hline 23 & 6 & 0 & 6.884466 & 1.493443 & 5721 \\
\hline 24 & 8 & 0 & -3.663543 & 0.831649 & 2.571741 \\
\hline 25 & 6 & 0 & -3.783831 & 1.835004 & 0.027557 \\
\hline 26 & 6 & 0 & -3.550425 & 1.787235 & -1.354389 \\
\hline 27 & 6 & 0 & -3.881903 & 2.873292 & -2.163750 \\
\hline 28 & 6 & 0 & -4.451359 & 4.019701 & -1.600720 \\
\hline 29 & 6 & 0 & -4.685853 & 4.074580 & -0.226076 \\
\hline 30 & 6 & 0 & -4.352380 & 2.986476 & 0.584703 \\
\hline 31 & 1 & 0 & -0.149096 & 1.273585 & 1.226416 \\
\hline 32 & 1 & 0 & -6.311233 & -3.449413 & -0.527152 \\
\hline 33 & 1 & 0 & -6.339588 & -1.0 & 64 \\
\hline 34 & 1 & 0 & & 554 & 1.260298 \\
\hline 35 & 1 & 0 & 4.4 & 90 & 1.150311 \\
\hline 36 & 1 & 0 & 4.816756 & -1.8 & -1.034807 \\
\hline 37 & 1 & 0 & 2.417398 & -2.190149 & -0.914789 \\
\hline 38 & 1 & 0 & 6.800900 & -1.901006 & -0.064179 \\
\hline 39 & 1 & 0 & 9.230660 & -1.575732 & -0.222707 \\
\hline 40 & 1 & 0 & 10.201464 & 0.723146 & -0.233009 \\
\hline 41 & 1 & 0 & 8.666994 & 2.687643 & -0.111895 \\
\hline 42 & 1 & 0 & 6.233987 & 2.363241 & -0.016594 \\
\hline 43 & 1 & 0 & -3.105057 & 0.897919 & -1.792675 \\
\hline 44 & 1 & 0 & -3.694374 & 2.827965 & -3.234206 \\
\hline 45 & 1 & 0 & -4.708202 & 4.866898 & -2.233137 \\
\hline 46 & 1 & 0 & -5.125907 & 4.965882 & 0.216462 \\
\hline 47 & 1 & 0 & 4.520668 & 3.011742 & 1.657587 \\
\hline
\end{tabular}


Table S11. Cartesian coordinates for minimized structure of compound 5-red.

\begin{tabular}{|c|c|c|c|c|c|}
\hline \multirow{2}{*}{$\begin{array}{l}\text { Center } \\
\text { Number }\end{array}$} & \multicolumn{3}{|c|}{ Atomic Atomic } & \multicolumn{2}{|c|}{ Coordinates (Angstroms } \\
\hline & & Number & Type & $\mathrm{X}$ & 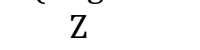 \\
\hline 1 & 15 & 0 & 0.000644 & 2.308721 & -1.180884 \\
\hline 2 & 6 & 0 & -1.297392 & 1.106805 & -0.765534 \\
\hline 3 & 6 & 0 & -0.702646 & -0.112278 & -0.352246 \\
\hline 4 & 6 & 0 & 0.704601 & -0.110586 & -0.347880 \\
\hline 5 & 6 & 0 & 1.299028 & 1.109556 & -0.758497 \\
\hline 6 & 6 & 0 & -2.693137 & 1.073204 & -0.758218 \\
\hline 7 & 6 & 0 & -3.243213 & -0.145207 & -0.351039 \\
\hline 8 & 16 & 0 & -1.925164 & -1.301774 & 0.036473 \\
\hline 9 & 16 & 0 & 1.927501 & -1.296950 & 0.049160 \\
\hline 10 & 6 & 0 & 3.245214 & -0.139601 & -0.337149 \\
\hline 11 & 6 & 0 & 2.694776 & 1.077936 & -0.746539 \\
\hline 12 & 6 & 0 & -4.627069 & -0.520396 & -0.226016 \\
\hline 13 & 6 & 0 & 4.629134 & -0.513698 & -0.209583 \\
\hline 14 & 6 & 0 & -5.675864 & 0.371491 & -0.576910 \\
\hline 15 & 6 & 0 & -7.007647 & 0.018119 & -0.449875 \\
\hline 16 & 6 & 0 & -7.403673 & -1.250821 & 0.027083 \\
\hline 17 & 6 & 0 & -6.367309 & 1697 & 0.370627 \\
\hline 18 & 6 & 0 & -5.0 & -1.793328 & 0.253286 \\
\hline 19 & 6 & 0 & 2 & 115 & 0.1 \\
\hline 20 & 6 & 0 & 6.3 & 311 & 08 \\
\hline 21 & 6 & 0 & 7.4 & 097 & 97 \\
\hline 22 & 6 & 0 & 7.00 & 0.049754 & -0.3 \\
\hline 23 & 6 & 0 & 5.677850 & 0.405222 & -0.48 \\
\hline 24 & 6 & 0 & -8.825368 & -1.622174 & 0.159633 \\
\hline 25 & 6 & 0 & 8.827624 & -1.614955 & 0.176541 \\
\hline 26 & 6 & 0 & -9.255072 & -2.956751 & 0.005916 \\
\hline 27 & 6 & 0 & -10.596730 & $0 \quad-3.307628$ & $\begin{array}{ll}8 & 0.136527\end{array}$ \\
\hline 28 & 6 & 0 & -11.560494 & $\begin{array}{ll}4 & -2.335798\end{array}$ & $8 \quad 0.417193$ \\
\hline 29 & 6 & 0 & -11.156640 & $0-1.007008$ & $8 \quad 0.568721$ \\
\hline 30 & 6 & 0 & -9.813762 & -0.657579 & 0.446141 \\
\hline 31 & 6 & 0 & 9.819003 & -1.027192 & -0.636674 \\
\hline 32 & 6 & 0 & 11.162165 & -1.374262 & $2-0.510955$ \\
\hline 33 & 6 & 0 & 55 & $5-2$ & 102 \\
\hline 34 & 6 & 0 & 10.5 & -2.9 & 1.23 \\
\hline 35 & 6 & 0 & 9.254993 & -2.572783 & 1.119668 \\
\hline 36 & 8 & 0 & 0.003337 & 2.979021 & -2.530756 \\
\hline 37 & 6 & 0 & -0.003986 & 3.585126 & 0.146673 \\
\hline 38 & 6 & 0 & -0.006718 & 3.253511 & 1.509141 \\
\hline 39 & 6 & 0 & -0.010278 & 4.256535 & 2.477532 \\
\hline 40 & 6 & 0 & -0.011165 & 5.601249 & 2.094297 \\
\hline 41 & 6 & 0 & -0.008475 & 5.937773 & 0.740028 \\
\hline 42 & 6 & 0 & -0.004889 & 4.932971 & -0.230757 \\
\hline 43 & 1 & 0 & -3.309595 & 1.918978 & -1.044557 \\
\hline 44 & 1 & 0 & 3.310956 & 1.921971 & -1.038586 \\
\hline 45 & 1 & 0 & -5.431943 & 1.354187 & -0.969416 \\
\hline 46 & 1 & 0 & -7.767012 & 0.730954 & -0.762584 \\
\hline 47 & 1 & 0 & -6.616259 & -3.120405 & 0.773944 \\
\hline 48 & 1 & 0 & -4.275348 & -2.515035 & 0.552419 \\
\hline
\end{tabular}




$\begin{array}{cccccc}49 & 1 & 0 & 4.277719 & -2.565667 & 0.402026 \\ 50 & 1 & 0 & 6.618757 & -3.177447 & 0.604243 \\ 51 & 1 & 0 & 7.768795 & 0.804301 & -0.553087 \\ 52 & 1 & 0 & 5.433819 & 1.420079 & -0.781821 \\ 53 & 1 & 0 & -8.526861 & -3.721923 & -0.247601 \\ 54 & 1 & 0 & -10.893421 & -4.346034 & 0.005051 \\ 55 & 1 & 0 & -12.608035 & -2.609335 & 0.515592 \\ 56 & 1 & 0 & -11.891099 & -0.237515 & 0.796846 \\ 57 & 1 & 0 & -9.515834 & 0.375379 & 0.602250 \\ 58 & 1 & 0 & 9.523689 & -0.308927 & -1.396347 \\ 59 & 1 & 0 & 11.898834 & -0.905083 & -1.159649 \\ 60 & 1 & 0 & 12.611594 & -2.601645 & 0.523255 \\ 61 & 1 & 0 & 10.891420 & -3.664061 & 1.982885 \\ 62 & 1 & 0 & 8.524448 & -3.023479 & 1.785545 \\ 63 & 1 & 0 & -0.006023 & 2.209135 & 1.809960 \\ 64 & 1 & 0 & -0.012347 & 3.990458 & 3.531926 \\ 65 & 1 & 0 & -0.013921 & 6.382562 & 2.851063 \\ 66 & 1 & 0 & -0.009142 & 6.982805 & 0.437998 \\ 67 & 1 & 0 & -0.002720 & 5.174376 & -1.289805 \\ ---------------------------------------------------------\end{array}$


Figure S12. ${ }^{1} \mathrm{H}$ NMR spectrum of 4 in $\mathrm{CDCl}_{3}$.

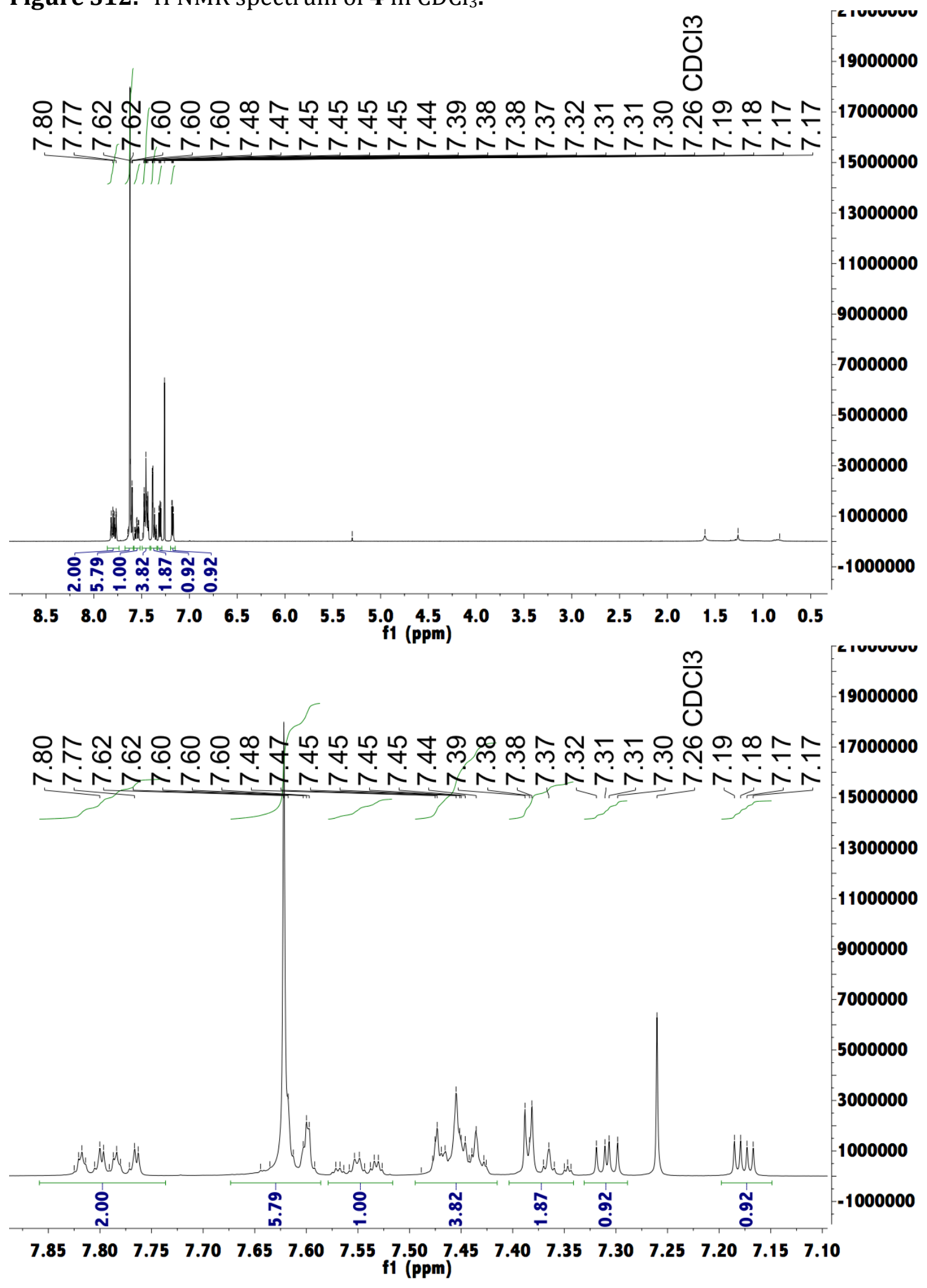


Figure S13. ${ }^{13} \mathrm{C}\left\{{ }^{1} \mathrm{H}\right\}$ APT NMR spectrum of 4 in $\mathrm{CDCl}_{3}$.

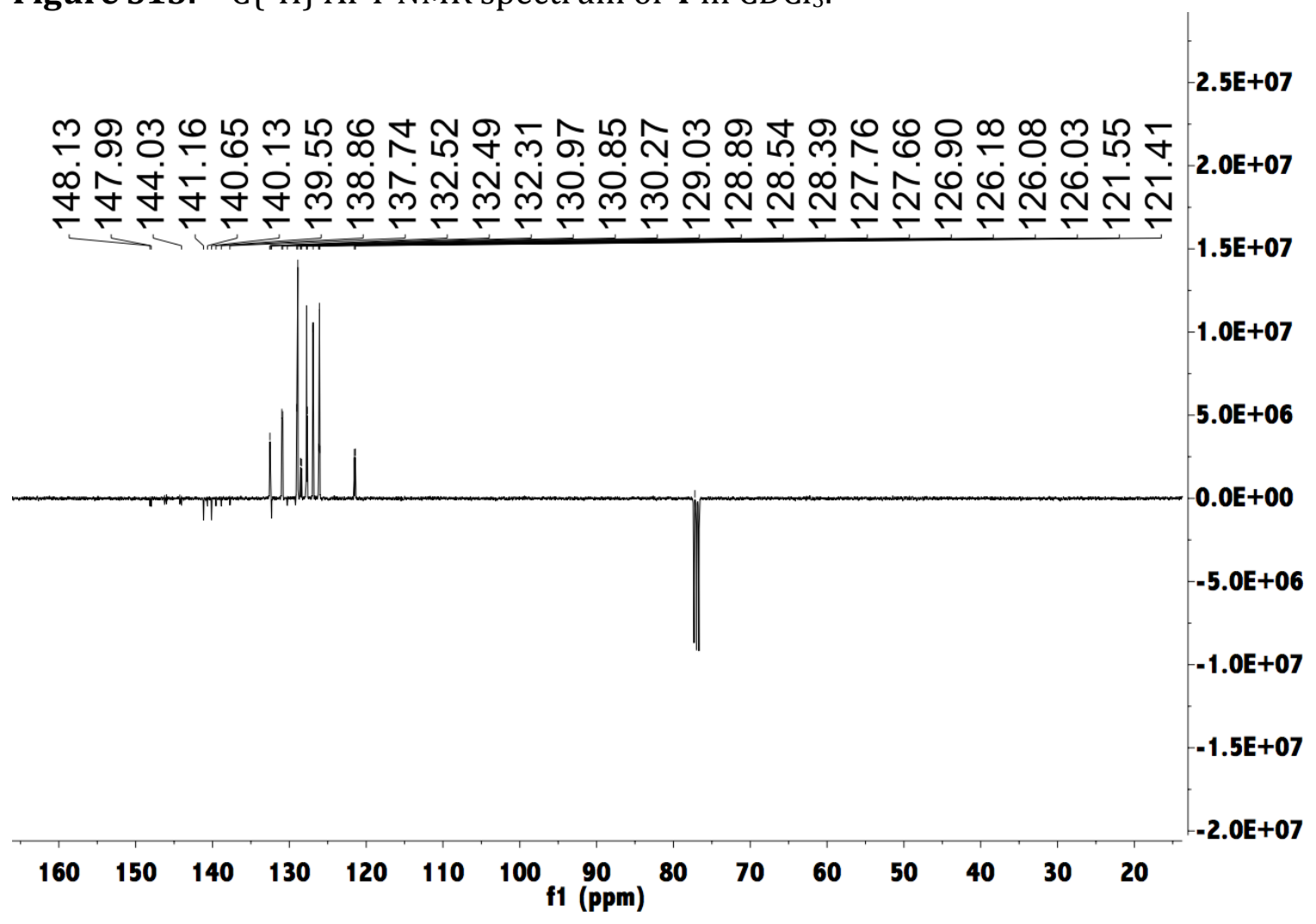

Figure S14. ${ }^{31} \mathrm{P}\left\{{ }^{1} \mathrm{H}\right\}$ NMR spectrum of 4 in $\mathrm{CDCl}_{3}$.

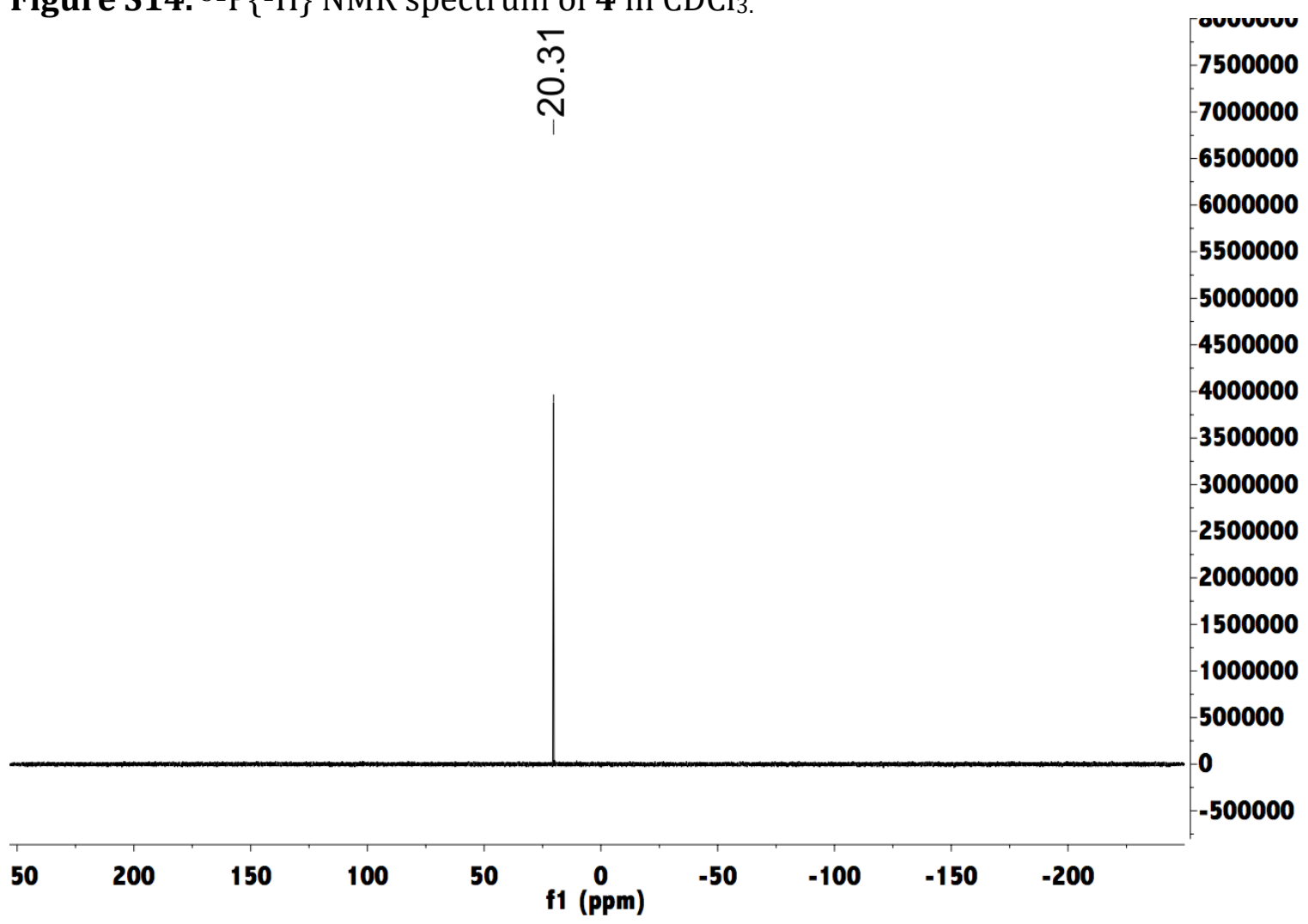


Figure S15. ${ }^{1} \mathrm{H}$ NMR spectrum of 5 in $\mathrm{CDCl}_{3}$.

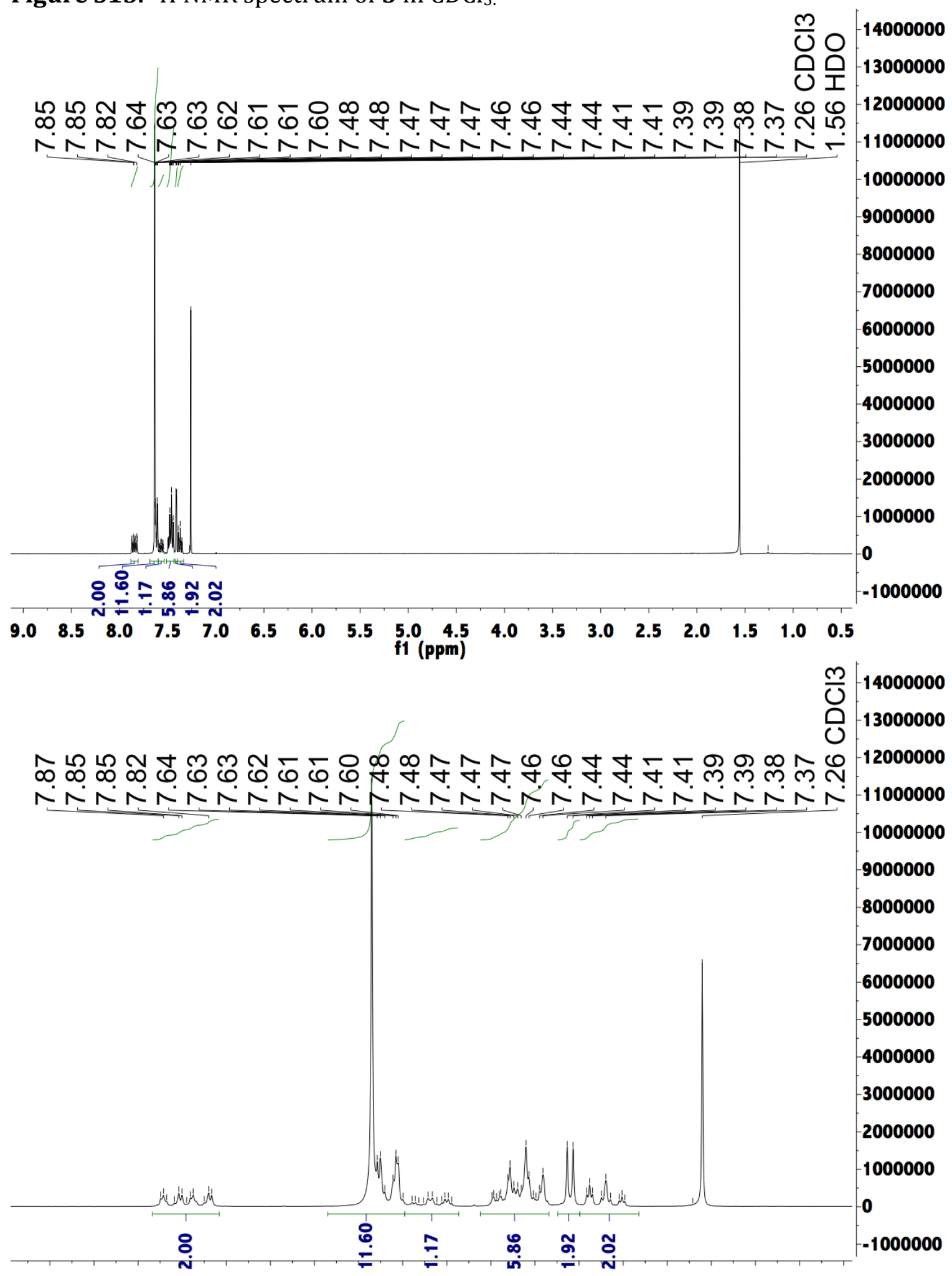

8.00 7.95 7.90 7.85 7.80 7.75 7.70 7.65 7.60 7.55 7.50 7.45 7.40 7.35 7.30 7.25 7.20 7.15 7.10 f1 (ppm) 
Figure S16. ${ }^{13} \mathrm{C}\left\{{ }^{1} \mathrm{H}\right\}$ APT NMR spectrum of $\mathbf{5}$ in $\mathrm{CDCl}_{3}$.

సิ

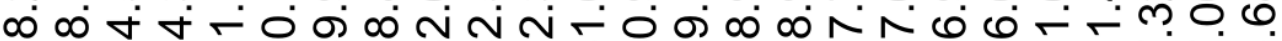

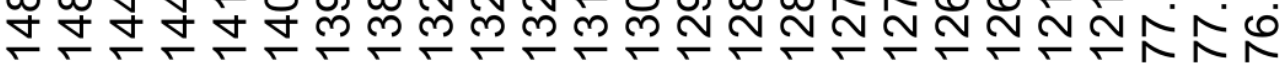

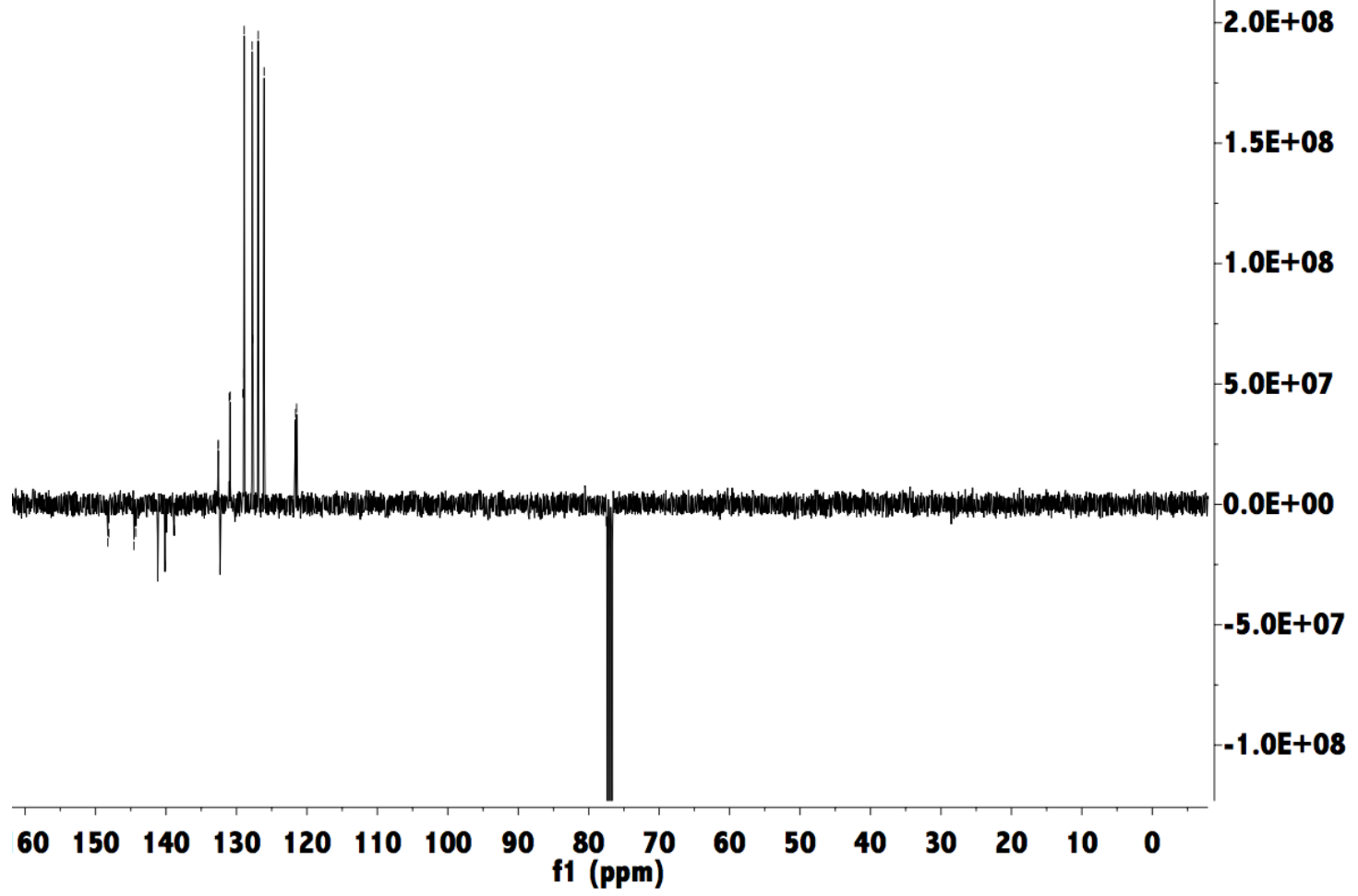

Figure S17. ${ }^{31} \mathrm{P}\left\{{ }^{1} \mathrm{H}\right\}$ NMR spectrum of $\mathbf{5}$ in $\mathrm{CDCl}_{3}$.

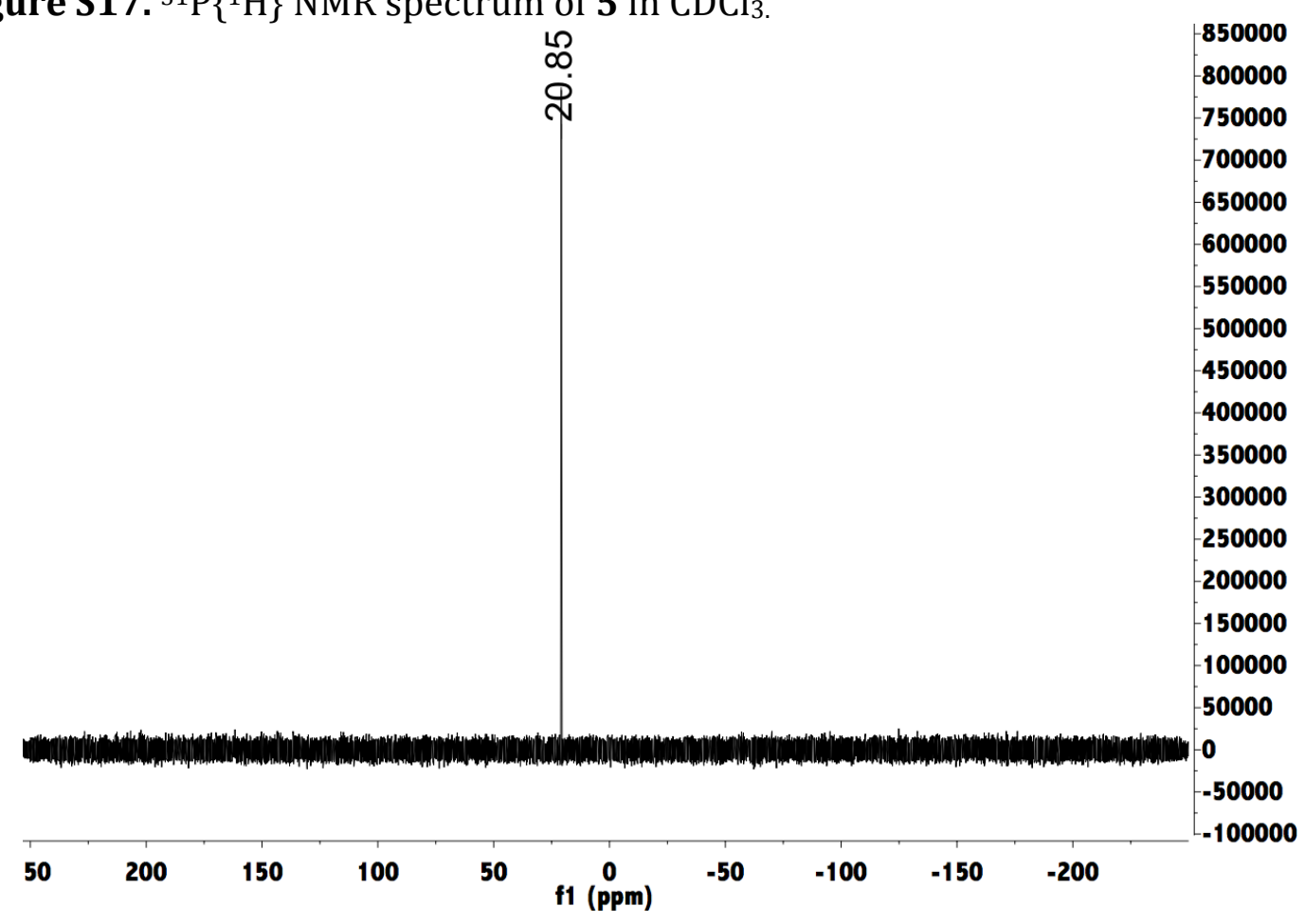


Figure S18. ${ }^{1} \mathrm{H}$ NMR spectrum of 6 in $\mathrm{CDCl}_{3}$.

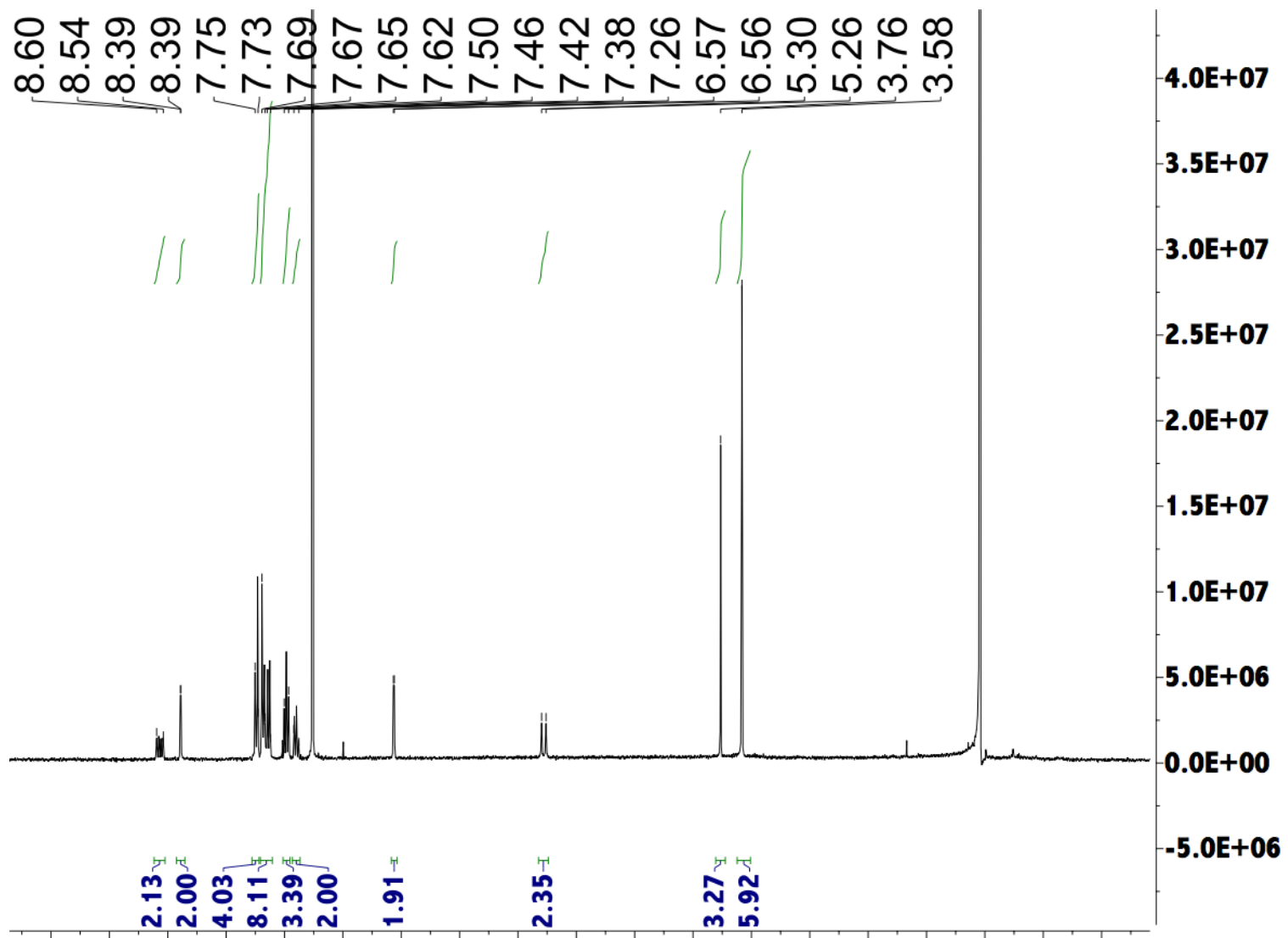

$\begin{array}{lllllllllllllllllll}9.5 & 9.0 & 8.5 & 8.0 & 7.5 & 7.0 & 6.5 & 6.0 & 5.5 & 5.0 & 4.5 & 4.0 & 3.5 & 3.0 & 2.5 & 2.0 & 1.5 & 1.0 & 0.5\end{array}$ f1 (ppm) 
Figure S19. ${ }^{13} \mathrm{C}\left\{{ }^{1} \mathrm{H}\right\}$ APT NMR spectrum of 6 in $\mathrm{CDCl}_{3}$.

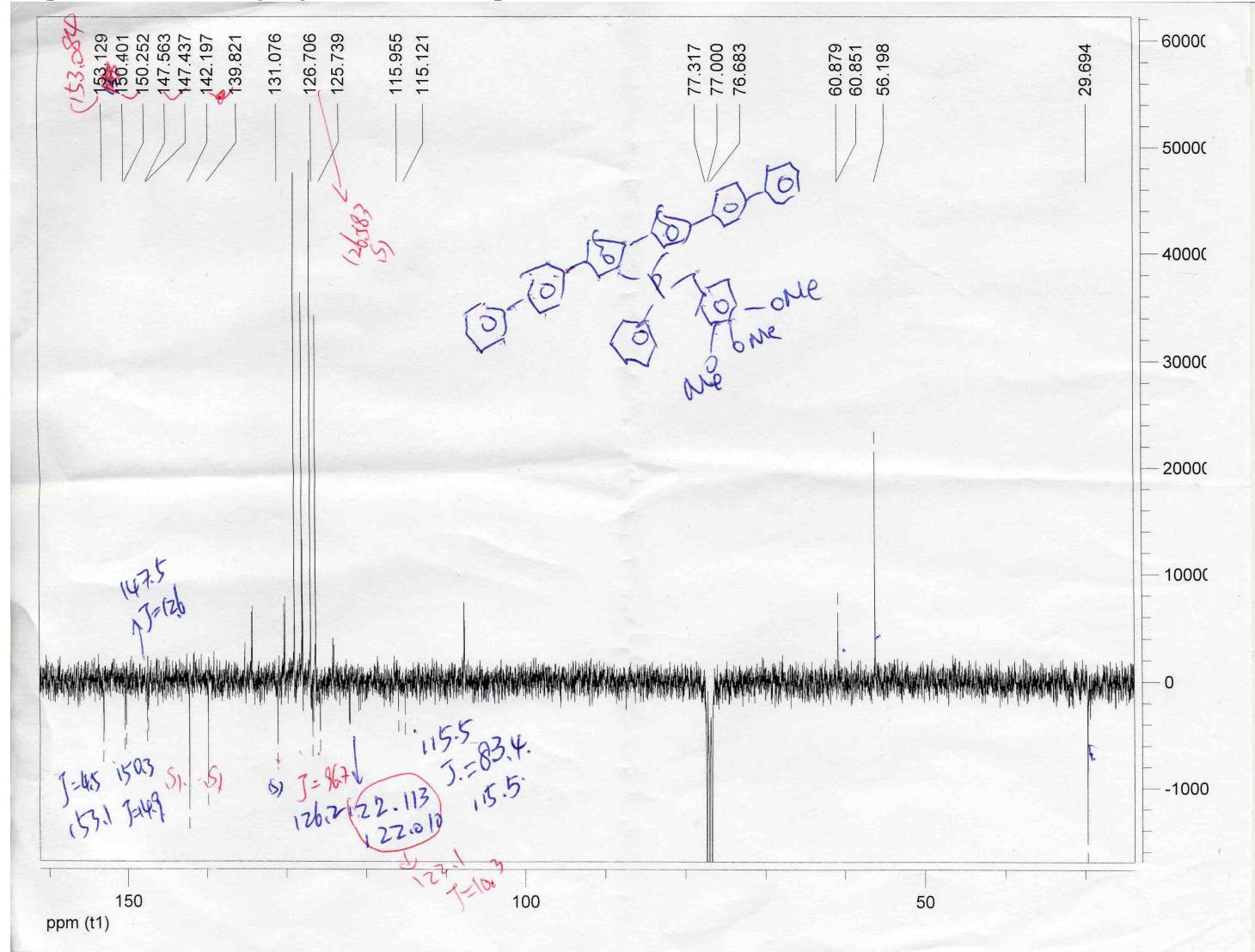

Figure S20. ${ }^{31} \mathrm{P}\left\{{ }^{1} \mathrm{H}\right\}$ NMR spectrum of 6 in $\mathrm{CDCl}_{3}$.

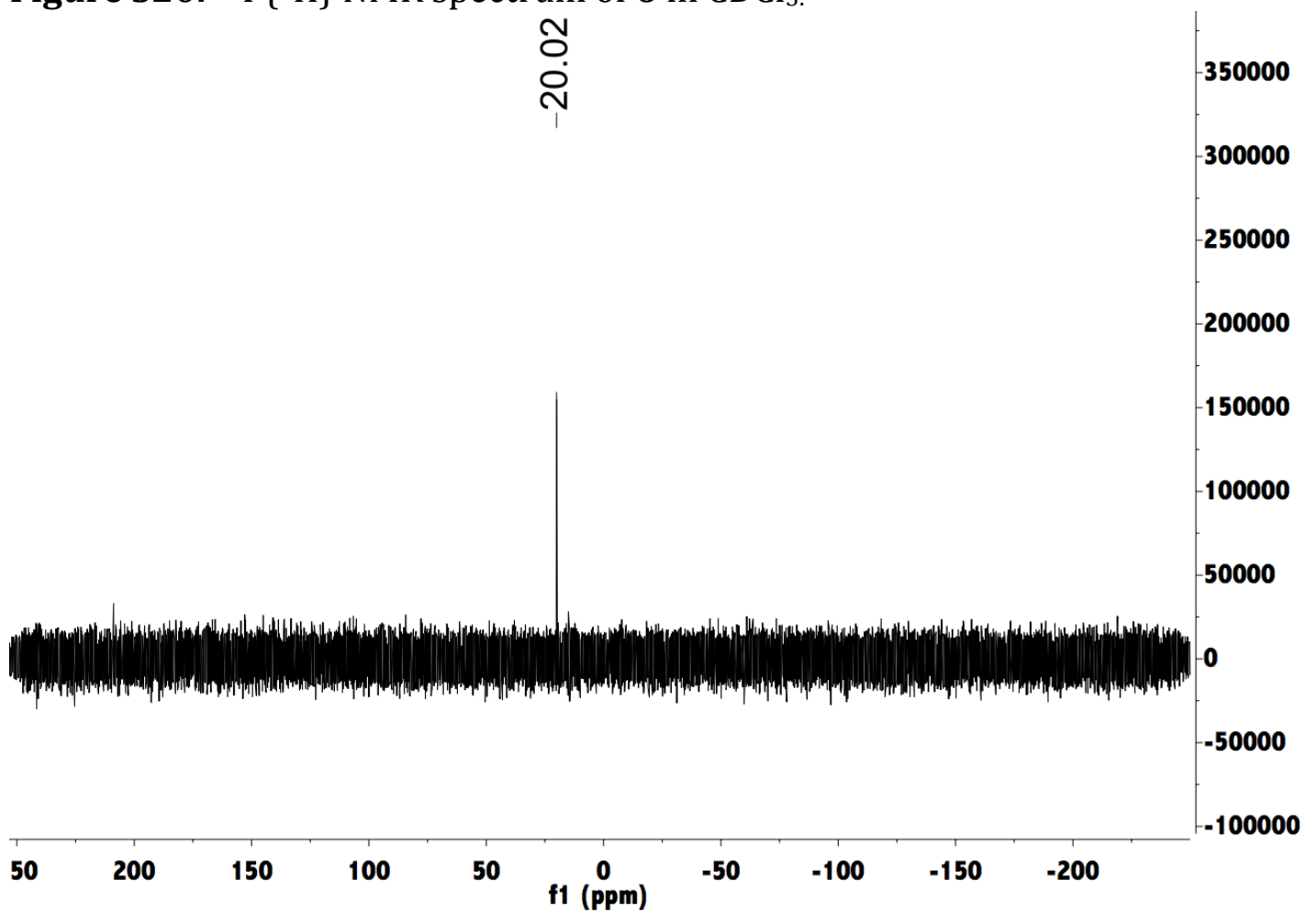

